\title{
Benzylic Substitution of Gramines with Boronic Acids and Rhodium or Iridium Catalysts
}

\author{
Gabriela de la Herrán, Amaya Segura, and Aurelio G. Csákÿ* \\ Departamento de Química Orgánica, Facultad de Química, Universidad Complutense. \\ 28040-Madrid, Spain. \\ E-mail: csaky@quim.ucm.es
}

\section{Supplementary material}

General procedures and copies of ${ }^{1} \mathrm{H}$ and ${ }^{13} \mathrm{C}-\mathrm{NMR}$ spectra

(34 pages)

General procedures............................................. 4

Copies of ${ }^{1} \mathrm{H}$ NMR and ${ }^{13} \mathrm{C}$ NMR spectra.............................S5-S34 


\section{General Methods}

All starting boronic acids were commercially available research-grade chemicals, and were used without further purification. The $\operatorname{Rh}(\mathrm{I})$ and $\operatorname{Ir}(\mathrm{I})$-catalysts were commercially available. Research-grade dioxane was used without further purification. Deionized water was used as co-solvent. Silica gel 60 F254 was used for TLC, and the spots were detected with UV or vanillin solution. Flash column chromatography was carried out on silica gel $60 .{ }^{1} \mathrm{H}-\mathrm{NMR}$ spectra were recorded at 200 $\mathrm{MHz}, 300 \mathrm{MHz}$ or $500 \mathrm{MHz}$ as indicated, in $\mathrm{CDCl}_{3}$ solution. ${ }^{13} \mathrm{C}$-NMR spectra were recorded at $50.5 \mathrm{MHz}$ or $75.5 \mathrm{MHz}$ as indicated in $\mathrm{CDCl}_{3}$ solution.

\section{Iridium-catalyzed synthesis of non-symmetical diindolylmethanes; General Procedure:}

To a mixture of $1(0.20 \mathrm{mmol})$ and $7(49.0 \mathrm{mg}, 0.25 \mathrm{mmol})$ under argon was added $[(\mathrm{cod}) \mathrm{IrCl}]_{2}(\mathbf{3 c} ; 7 \mathrm{mg}, 0.01 \mathrm{~mol}), \mathrm{K}_{3} \mathrm{PO}_{4}(42.4 \mathrm{mg}, 0.2 \mathrm{mmol})$ and dioxane- $\mathrm{H}_{2} \mathrm{O}$ $(10: 1,1.0 \mathrm{~mL})$. The reaction mixture was stirred at $50^{\circ} \mathrm{C}$ for $18 \mathrm{~h}$. Products were isolated by evaporation of volatiles under reduced pressure followed by column chromatography (75:25, hexane - ethyl acetate).

\section{Rhodium-catalyzed synthesis of 3-benzyl and 3-allylindoles; General Procedure:}

To a mixture of $8(0.20 \mathrm{mmol})$ and $2(0.40 \mathrm{mmol})$ under argon was added $\left[(\operatorname{cod})_{2} \mathrm{Rh}\right] \mathrm{BF}_{4}(3 \mathbf{b} ; 4 \mathrm{mg}, 0.01 \mathrm{~mol}), \mathrm{K}_{3} \mathrm{PO}_{4}(42.4 \mathrm{mg}, 0.2 \mathrm{mmol})$ and dioxane- $\mathrm{H}_{2} \mathrm{O}$ $(8: 2,1.0 \mathrm{~mL})$. The reaction mixture was stirred at $65^{\circ} \mathrm{C}$ for $18 \mathrm{~h}$. Products were isolated by evaporation of volatiles under reduced pressure followed by column chromatography (85:15, hexane - ethyl acetate). 


\section{5-Bromo-3,3'-diindolylmethane, 6a}

${ }^{1} \mathrm{H}$ NMR $\left(200 \mathrm{MHz}, \mathrm{CDCl}_{3}\right): \delta=7.78$ (br s, 2H), 7.65 (br s, 1H), 7.51 (d, J=8.1 Hz, 1H), 7.21-7.06 (m, 4H), 7.01 (br t, J=7.5 Hz, 1H), 6.80 (br s, 1H), 4.08 (s, 2H); ${ }^{33} \mathrm{C} \mathrm{NMR}\left(50 \mathrm{MHz}, \mathrm{CDCl}_{3}\right)$ : $\delta=136.9,135.5,129.7,127.8,125.2,123.9,122.6,122.4,122.3,122.2,122.1,119.7,115.8$, 115.2, 113.0, 111.6, 21.5. Anal. Calcd. For $\mathrm{C}_{17} \mathrm{H}_{13} \mathrm{BrN}_{2}$ : $\mathrm{C}, 62,79 ; \mathrm{H}, 4$,03.Found: $\mathrm{C}, 62.84 ; \mathrm{H}$, 4.17

\section{5-Bromo-5'-methoxy-3,3'-diindolylmethane, 6b}

${ }_{1}^{1} \mathrm{H} \mathrm{NMR}\left(200 \mathrm{MHz}, \mathrm{CDCl}_{3}\right): \delta=7.95$ (br s, $\left.1 \mathrm{H}\right), 7.84$ (br s, 1H), 7.72 (br s, $\left.1 \mathrm{H}\right), 7.31-716(\mathrm{~m}, 3 \mathrm{H})$, 7.09-7.01 (m, 1H), 6.94-6.87 (m, 2H), $6.85(\mathrm{~d}, \mathrm{~J}=3.0 \mathrm{~Hz}, 1 \mathrm{H}), 4.15(\mathrm{~s}, 2 \mathrm{H}), 3.82(\mathrm{~s}, 3 \mathrm{H}) ;{ }^{13 \mathrm{C}} \mathrm{NMR}$ $\left(50 \mathrm{MHz}, \mathrm{CDCl}_{3}\right): \delta=153.9,135.0,131.6,129.3,127.8,124.7,123.4,123.0,121.8,115.3,114.8$, $112.5,112.1,111.8,101.1,55.9,21.1$. Anal. Calcd. For $\mathrm{C}_{18} \mathrm{H}_{15} \mathrm{BrN}_{2} \mathrm{O}: \mathrm{C}, 60,86 ; \mathrm{H}, 4,26$.Found: C, $60.98 ; \mathrm{H}, 4.35$.

\section{6-Bromo-5'-methoxy-3,3'-diindolylmethane, 6c}

${ }^{1} \mathrm{H} \mathrm{NMR}\left(200 \mathrm{MHz}, \mathrm{CDCl}_{3}\right): \delta=7.99$ (br s, 1H), 7.82 (br s, 1H), 7.75 (br s, 1H), $7.43(\mathrm{~d}, \mathrm{~J}=8.51 \mathrm{H})$, 7.29-7.19 (m, 2H), 6.95 (br s, 1H), 6.89-6.80 (m, 1H), 6.75 (dd, J=8.5, 2.0, 1H), $4.15(\mathrm{~s}, 2 \mathrm{H}), 3.85$ (s, 3H). ${ }^{13} \mathrm{C}$ NMR $\left(50 \mathrm{MHz}, \mathrm{CDCl}_{3}\right): \delta=156.4,137.1,135.1,129.2,124.7,124.6,121.8,121.6$, $120.9,119.6,115.3,115.0,114.7,112.4,109.1,94.7,55.6,21.1$. Anal. Calcd. For $\mathrm{C}_{18} \mathrm{H}_{15} \mathrm{BrN}_{2} \mathrm{O}$ : C, 60,86; H, 4,26.Found: C, 60.95; H, 4.39.

\section{3-Benzyl-1H-indole, 5a}

${ }^{1} \mathrm{H} \mathrm{NMR}\left(200 \mathrm{MHz}, \mathrm{CDCl}\right.$ ): $\delta=7.85$ (br s, 1H), 7.32-6.99 (m, 8H), 6.82 (br s, 1H), 4.07 (s, 2H); ${ }^{13} \mathrm{C}$ $\operatorname{NMR}\left(50 \mathrm{MHz}, \mathrm{CDCl}_{3}\right): \delta=141.3,136.3,128.7,128.4,125.9,122.4,122.1,119.4,115.6,119.2$, 111.1, 31.6. Anal. Calcd. For $\mathrm{C}_{15} \mathrm{H}_{13} \mathrm{~N}$ : C, 86,92; $\mathrm{H}, 6,32$. Found: $\mathrm{C}, 87.03 ; \mathrm{H}, 6.21$.

\section{3-(4-Methoxybenzyl)-1H-indole, 5b}

${ }^{1} \mathrm{H}$ NMR $\left(200 \mathrm{MHz}, \mathrm{CDCl}_{3}\right): \delta=7.82$ (br s, 1H), 7.44 (d, J=7.5 Hz, 1H), 7.25 (d, J=7.5 Hz, 1H), 7.16-6.92 (m, 4H), 6.82-6.71 (m, 3H), 3.97 (s, 2H), 3.69 (s, 3H); ${ }^{13} \mathrm{C}$ NMR (50 MHz, $\left.\mathrm{CDCl}_{3}\right)$ : $\delta=157.9,136.6,133.4,129.7,127.6,122.3,122.1,119.4,119.3,116.4,113.9,111.4,55.4,30.8$. Anal. Calcd. For $\mathrm{C}_{16} \mathrm{H}_{15} \mathrm{NO}$ : C, 80,98; $\mathrm{H}, 6$,37.Found: $\mathrm{C}, 81.07 ; \mathrm{H}, 6.44$.

\section{3-(2-Methoxybenzyl)-1H-indole, 5c}

${ }^{1} \mathrm{H}$ NMR $\left(200 \mathrm{MHz}, \mathrm{CDCl}_{3}\right): \delta=7.80$ (br s, 1H), 7.49 (d, J=7.5 Hz, 1H), 7.23 (d, J=7.5 Hz, 1H), 7.14-6.91 (m, 4H), 6.82 (br s, 1H), 6.82-6.70 (m, 2H), 4.02 (s, 2H), $3.78(\mathrm{~s}, 3 \mathrm{H}) ;{ }^{13} \mathrm{C}$ NMR $(50$ $\left.\mathrm{MHz}, \mathrm{CDCl}_{3}\right): \delta=157.1,136.2,129.7,129.5,127.6,126.9,122.2,121.6,120.2,119.1,119.0$, 115.1, 110.8, 110.0, 55.2, 24.9. Anal. Calcd. For $\mathrm{C}_{16} \mathrm{H}_{15} \mathrm{NO}$ : C, 80,98; H, 6,37.Found: $\mathrm{C}, 81.22 ; \mathrm{H}$, 6.51.

\section{3-(4-Trifluorobenzyl)-1H-indole, 5d}

${ }^{1} \mathrm{H}$ NMR $\left(500 \mathrm{MHz}, \mathrm{CDCl}_{3}\right): \delta=8.03(\mathrm{br} \mathrm{s}, 1 \mathrm{H}), 7.67-7.63(\mathrm{~m}, 2 \mathrm{H}), 7.57-7.51(\mathrm{~m}, 1 \mathrm{H}), 7.49-7.37$ $(\mathrm{m}, 3 \mathrm{H}), 7.38-7.35(\mathrm{~m}, 1 \mathrm{H}), 7.19-7.12(\mathrm{~m}, 1 \mathrm{H}), 6.98(\mathrm{br} \mathrm{s}, 1 \mathrm{H}), 4.05(\mathrm{~s}, 2 \mathrm{H}) ;{ }^{13} \mathrm{C}$ NMR $(125 \mathrm{MHz}$, $\left.\mathrm{CDCl}_{3}\right): \delta=145.8,136.9,129.3,128.6,127.6,125.7,125.6,122.9,122.7,120.0,119.4,115.1$, 111.6, 31.9. Anal. Calcd. For $\mathrm{C}_{16} \mathrm{H}_{12} \mathrm{~F}_{3} \mathrm{~N}$ : C, 69,81; $\mathrm{H}, 4,39$.Found: $\mathrm{C}, 70.03 ; \mathrm{H}, 4.56$.

\section{5-Methoxy-3-(4-trifluorobenzyl)-1H-indole, 5e}

${ }^{1} \mathrm{H}$ NMR $\left(500 \mathrm{MHz}, \mathrm{CDCl}_{3}\right): \delta=7.96$ (br s, $\left.1 \mathrm{H}\right), 7.57-7.55$ (m, 2H), 7.42-7.41 (m, 2H), 7.30-7.25 $(\mathrm{m}, 1 \mathrm{H}), 6.98-6.88(\mathrm{~m}, 3 \mathrm{H}), 4.17(\mathrm{~s}, 2 \mathrm{H}), 3.72(\mathrm{~s}, 3 \mathrm{H}) ;{ }^{13} \mathrm{C}$ NMR (125 MHz, $\left.\mathrm{CDCl}_{3}\right): \delta=154.5$, 
145.7, 132.0, 129.3, 128.5, 128.1, 125.9, 125.7, 123.9, 114.8, 112.6, 112.5, 101.4, 56.4, 31.9. Anal. Calcd. For $\mathrm{C}_{17} \mathrm{H}_{14} \mathrm{~F}_{3} \mathrm{NO}$ : C, 66,88; $\mathrm{H}, 4,62$.Found: C, 67.10; H, 4.77.

\section{6-Methoxy-3-(4-trifluorobenzyl)-1H-indole, $5 f$}

${ }^{1} \mathrm{H}$ NMR $\left(500 \mathrm{MHz}, \mathrm{CDCl}_{3}\right): \delta=7.93$ (br s, 1H), 7.56-7.47 (m, 2H), 7.40 (m. 2H), $7.35(\mathrm{~m}, 1 \mathrm{H})$, 6.92-6.84 (m, 2H), $6.78(\mathrm{~m}, 1 \mathrm{H}), 4.16(\mathrm{~s}, 2 \mathrm{H}), 3.80(\mathrm{~s}, 3 \mathrm{H}) ;{ }^{13} \mathrm{C} \mathrm{NMR}\left(125 \mathrm{MHz}, \mathrm{CDCl}_{3}\right): \delta=157.1$, 145.8, 137.7, 129.2, 128.7, 125.8, 123.7, 122.1, 121.7, 119.8, 115.0, 109.9, 95.2, 56.1, 31.3. Anal. Calcd. For $\mathrm{C}_{17} \mathrm{H}_{14} \mathrm{~F}_{3} \mathrm{NO}$ : C, 66,88; $\mathrm{H}, 4,62$. Found: $\mathrm{C}, 67.07 ; \mathrm{H}, 4.81$.

\section{3-(2-Bromobenzyl)-1H-indole, $5 \mathrm{~g}$}

${ }^{1} \mathrm{H} \mathrm{NMR}\left(200 \mathrm{MHz}, \mathrm{CDCl}_{3}\right): \delta=7.83$ (br s, 1H), 7.51-7.43 (t, J=7.3 Hz, 2H), 7.26 (d, J = 7.6 Hz, $1 \mathrm{H}), 7.18-6.89(\mathrm{~m}, 5 \mathrm{H}), 6.79(\mathrm{br} \mathrm{s}, 1 \mathrm{H}), 4.12(\mathrm{~s}, 2 \mathrm{H}) ;{ }^{13} \mathrm{C}$ NMR $\left(50 \mathrm{MHz}, \mathrm{CDCl}_{3}\right): \delta=140.6,136.6$, 132.8, 130.8, 127.8, 127.6, 124.8, 123.0, 122.3, 119.7, 119.4, 114.2, 111.4, 32.0. Anal. Calcd. For $\mathrm{C}_{15} \mathrm{H}_{12} \mathrm{BrN}$ : C, 62,96; $\mathrm{H}, 4,23$. Found: $\mathrm{C}, 63.13 ; \mathrm{H}, 4.04$.

\section{3-(3-Phenylallyl)-1H-indole, 9a}

${ }^{1} \mathrm{H}$ NMR $\left(200 \mathrm{MHz}, \mathrm{CDCl}_{3}\right): \delta=7.89(\mathrm{br} \mathrm{s}, 1 \mathrm{H}), 7.61-7.56(\mathrm{~d}, \mathrm{~J}=7.6 \mathrm{~Hz}, 1 \mathrm{H}), 7.35-6.95(\mathrm{~m}, 9 \mathrm{H})$, 6.60-6.49 (m, 2H), 3.63 (d, J=5.5 Hz, 2H); ${ }^{13} \mathrm{C}$ NMR (50 MHz, $\left.\mathrm{CDCl}_{3}\right): \delta=137.3,136.8,130.5$, 129.3, 128.5, 127.1, 126.9, 126.1, 122.1, 121.8, 119.4, 119.2, 111.1, 28.9. Anal. Calcd. For $\mathrm{C}_{17} \mathrm{H}_{15} \mathrm{~N}: \mathrm{C}, 87,52 ; \mathrm{H}, 6,48$. Found: $\mathrm{C}, 87.69 ; \mathrm{H}, 6.36$.

\section{3-[3-(4-Trifluoromethylphenyl)allyl]-1H-indole, $9 \mathrm{~b}$}

${ }^{1} \mathrm{H}$ NMR (500 MHz, $\left.\mathrm{CDCl}_{3}\right): \delta=8.10$ (br s, 1H), $7.65(\mathrm{~m}, 1 \mathrm{H}), 7.55(\mathrm{~m}, 2 \mathrm{H}), 7.47(\mathrm{~m}, 2 \mathrm{H}), 7.43(\mathrm{~m}$, 2H), $7.25(\mathrm{~m}, 1 \mathrm{H}), 7.15(\mathrm{~m}, 1 \mathrm{H}), 7.04$ (br s, $1 \mathrm{H}), 6.62-6.51(\mathrm{~m} \mathrm{2H}), 3.75(\mathrm{~d}, \mathrm{~J}=4.5 \mathrm{~Hz}, 1 \mathrm{H}) ;{ }^{13} \mathrm{C}$ $\operatorname{NMR}\left(125 \mathrm{MHz}, \mathrm{CDCl}_{3}\right): \delta=141.6,136.8,132.6,129.6,127.7,126.6,125.9,125.8,122.6,122.3$, 119.9, 119.4, 114.4, 111.6, 29.4. Anal. Calcd. For $\mathrm{C}_{18} \mathrm{H}_{14} \mathrm{~F}_{3} \mathrm{~N}: 71,75 ; \mathrm{H}, 4,68$. Found: $\mathrm{C}, 71.92 ; \mathrm{H}$, 4.89 .

\section{3-[3-(4-Chlorophenyl)allyl]-1H-indole, $9 \mathrm{c}$}

${ }^{1} \mathrm{H}$ NMR $\left(500 \mathrm{MHz}, \mathrm{CDCl}_{3}\right): \delta=8.01$ (br s, $\left.1 \mathrm{H}\right), 7.65$ (d, J=7.5 Hz, 1H), 7.35 (d, J=7.5 Hz, 1H), 7.33-7.22 (m, 5H), $7.15(\mathrm{~m}, 1 \mathrm{H}), 7.06$ (br s, 1H), 6.53-6.44 (m, 2H), $3.71(\mathrm{~d}, \mathrm{~J}=4.8 \mathrm{~Hz}, 2 \mathrm{H}) ;{ }^{13} \mathrm{C}$ $\operatorname{NMR}\left(125 \mathrm{MHz}, \mathrm{CDCl}_{3}\right): \delta=136.8,136.6,132.9,131.3,130.7,129.7,129.3,128.0,127.8,122.5$, 122.1, 121.2, 119.8, 119.5, 114.7, 111.5, 28.9. Anal. Calcd. For $\mathrm{C}_{17} \mathrm{H}_{14} \mathrm{CIN}: 76,26 ; \mathrm{H}, 5,27$. Found: C, 76.52; H, 4.99.

\section{5-Methoxy-3-(3-phenylallyl)-1H-indole, $9 \mathrm{~d}$}

${ }^{1} \mathrm{H}$ NMR $\left(200 \mathrm{MHz}, \mathrm{CDCl}_{3}\right): \delta=7.81$ (br s, 1H), 7.32-7.07 (m, 6H), 6.97 (dd, J=12.1, $2.4 \mathrm{~Hz}, 2 \mathrm{H}$ ), 6.78 (dd, J=8.8, $2.4 \mathrm{~Hz}, 1 \mathrm{H}), 6.51-6.30(\mathrm{~m}, 2 \mathrm{H}), 3.77$ (s, 3H), 3.59 (d, J=5.5 Hz, 2H); ${ }^{13} \mathrm{C}$ NMR (50 $\left.\mathrm{MHz}, \mathrm{CDCl}_{3}\right): \delta=153.9,143.2,137.8,130.4,129.1,128.4,126.8,126.0,122.5,114.5,112.1$, 111.7, 101.0, 55.8, 28.9. Anal. Calcd. For $\mathrm{C}_{18} \mathrm{H}_{17} \mathrm{NO}$ : 82,10; $\mathrm{H}, 6,51$. Found: C, 82.23; $\mathrm{H}, 6.72$.

\section{6-Methoxy-3-(3-phenylallyl)-1H-indole, 9e}

${ }^{1} \mathrm{H}$ NMR $\left(200 \mathrm{MHz}, \mathrm{CDCl}_{3}\right): \delta=7.79$ (br s, 1H), 7.67-7.05 (m, 6H), 6.81 (dd, J=12.1, $\left.2.4 \mathrm{~Hz}, 2 \mathrm{H}\right)$, 6.71 (dd, J=8.8, $2.4 \mathrm{~Hz}, 1 \mathrm{H}), 6.51-6.29(\mathrm{~m}, 2 \mathrm{H}), 3.77$ (s, 3H), 3.58 (d, J=5.7 Hz, 2H); ${ }^{13} \mathrm{C}$ NMR (50 $\left.\mathrm{MHz}, \mathrm{CDCl}_{3}\right): \delta=157.1,138.3,137.2,130.5,129.4,128.5,127.0,126.1,120.5,119.8,114.7$, 109.4, 94.8, 55.8, 29.8. Anal. Calcd. For $\mathrm{C}_{18} \mathrm{H}_{17} \mathrm{NO}$ : 82,10; H, 6,51. Found: C, 82.25; H, 6.74. 


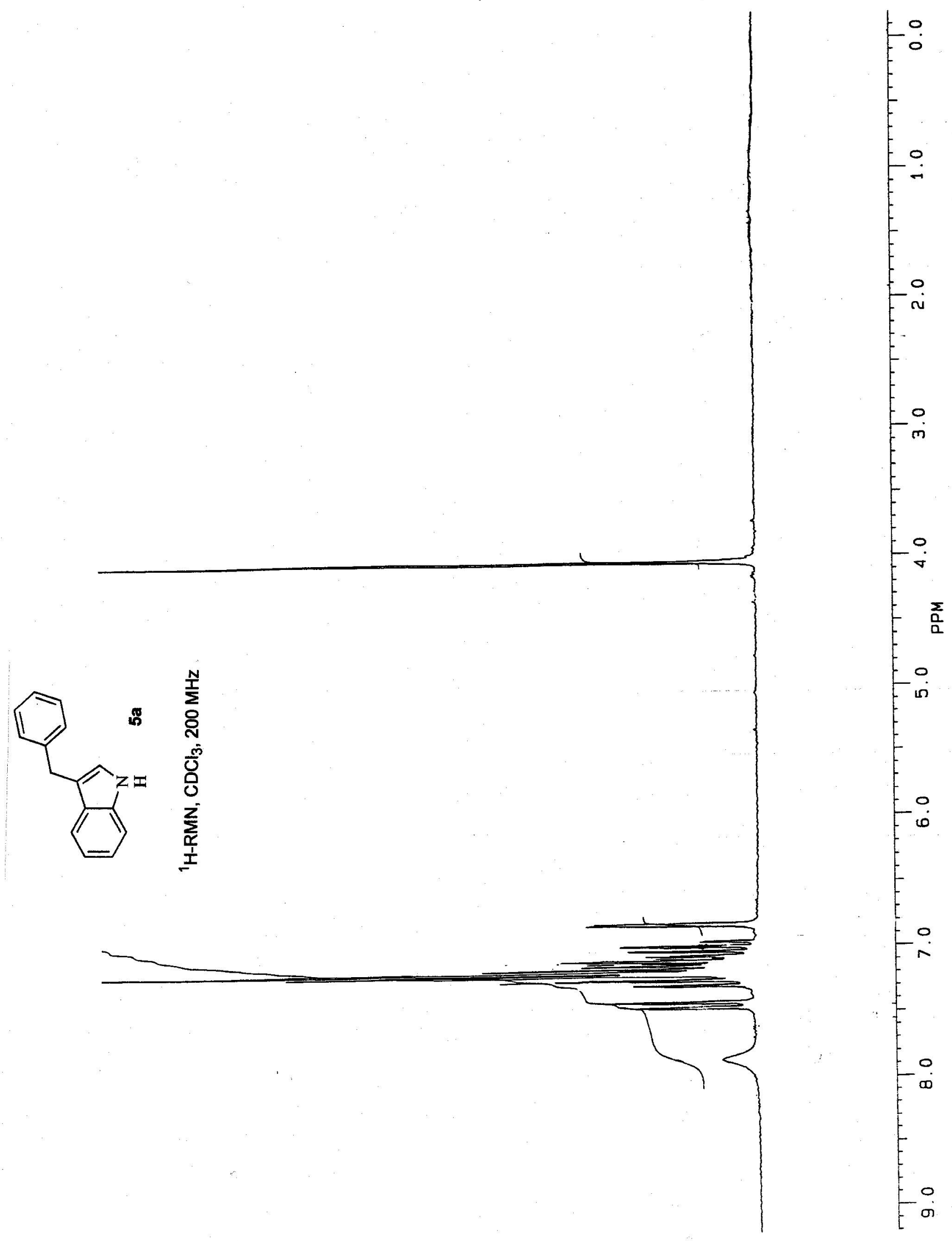




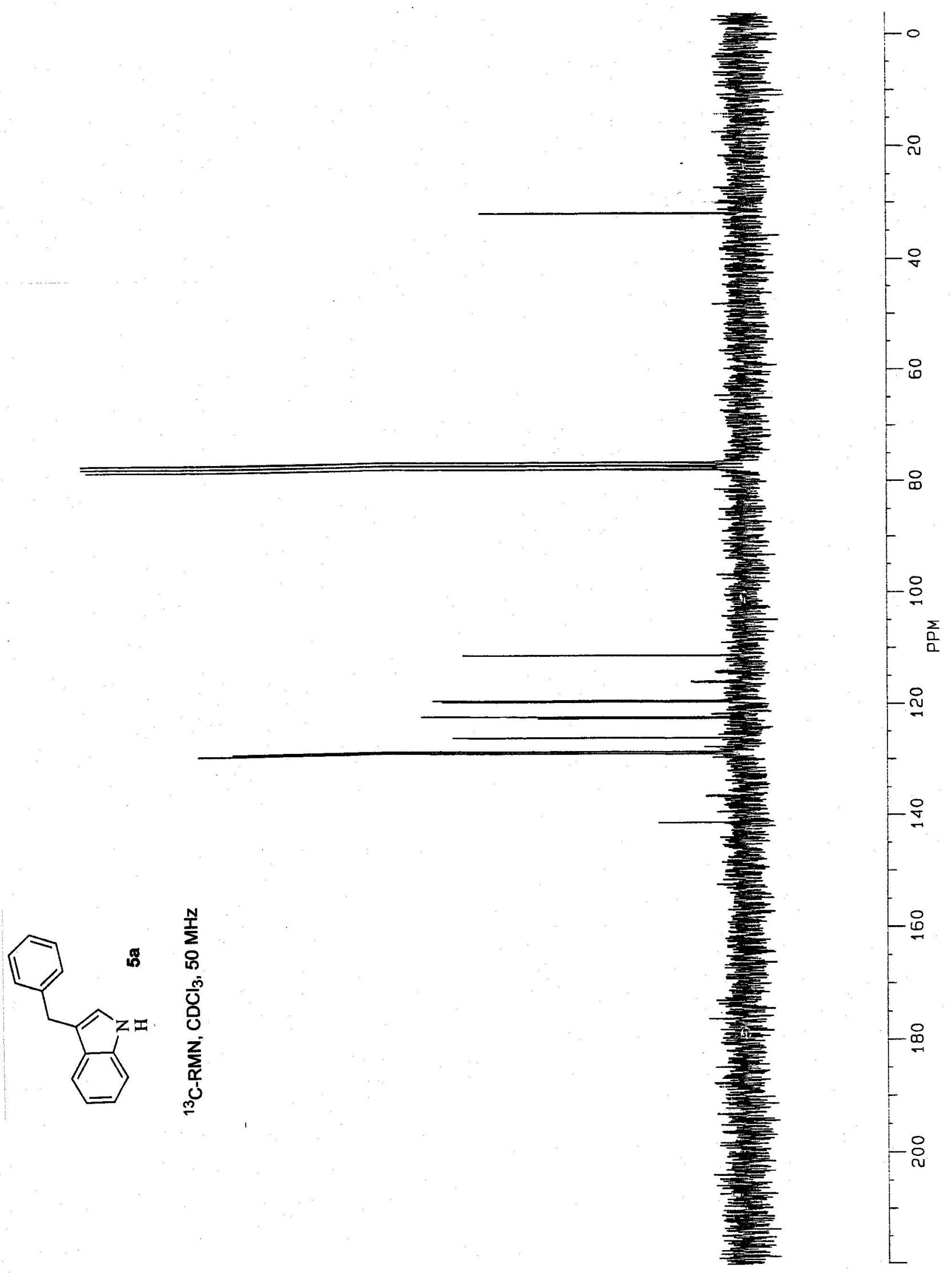




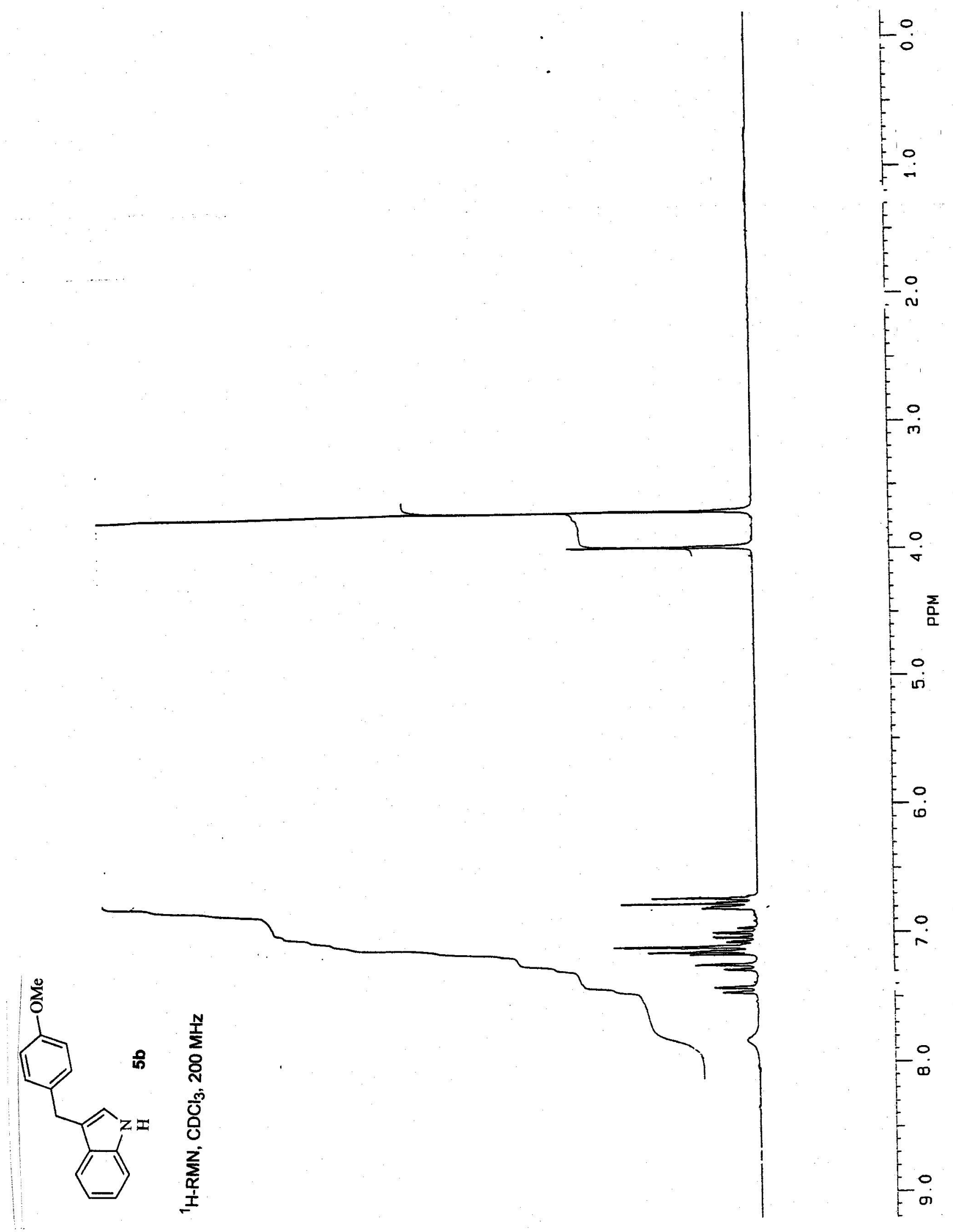




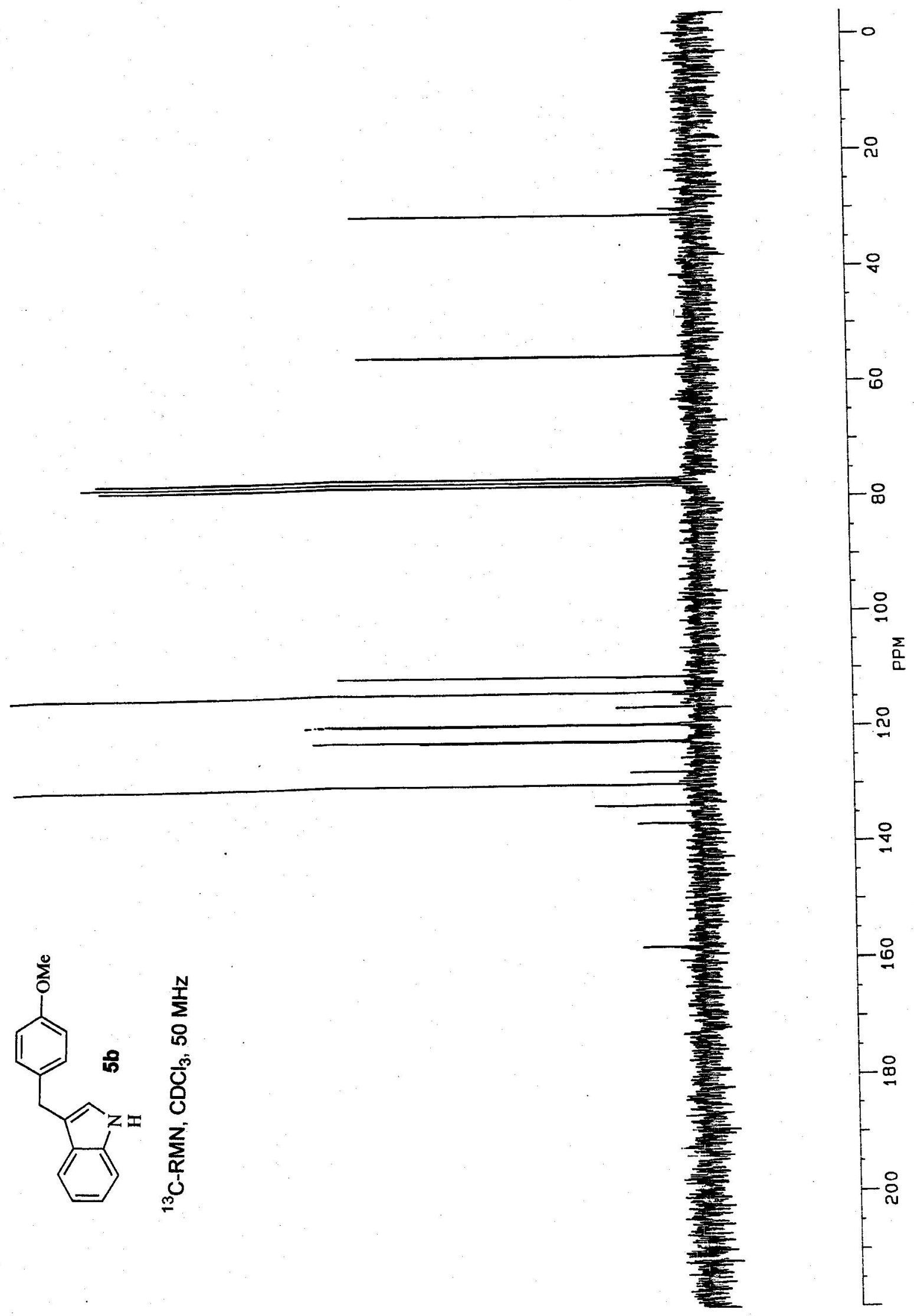




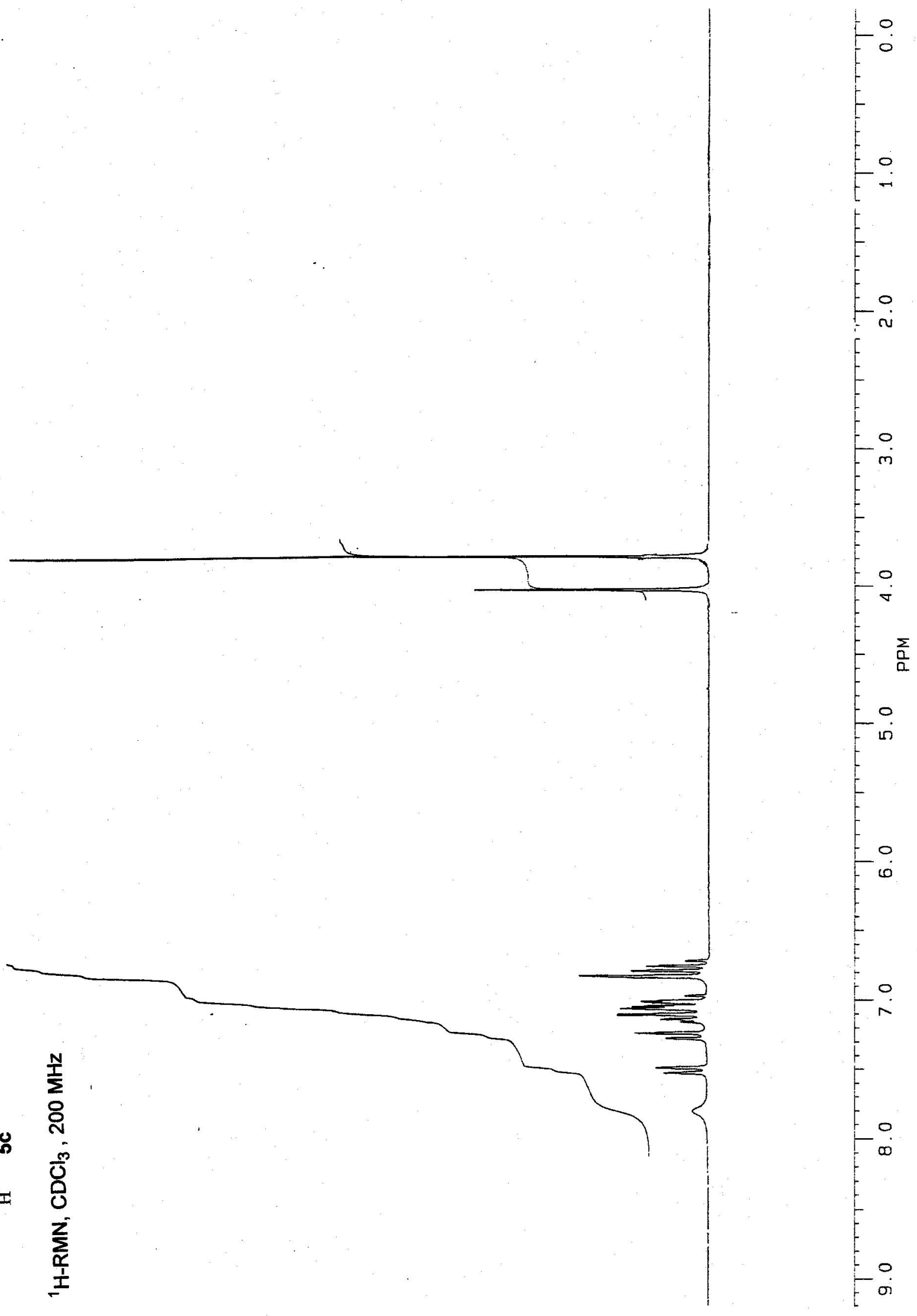




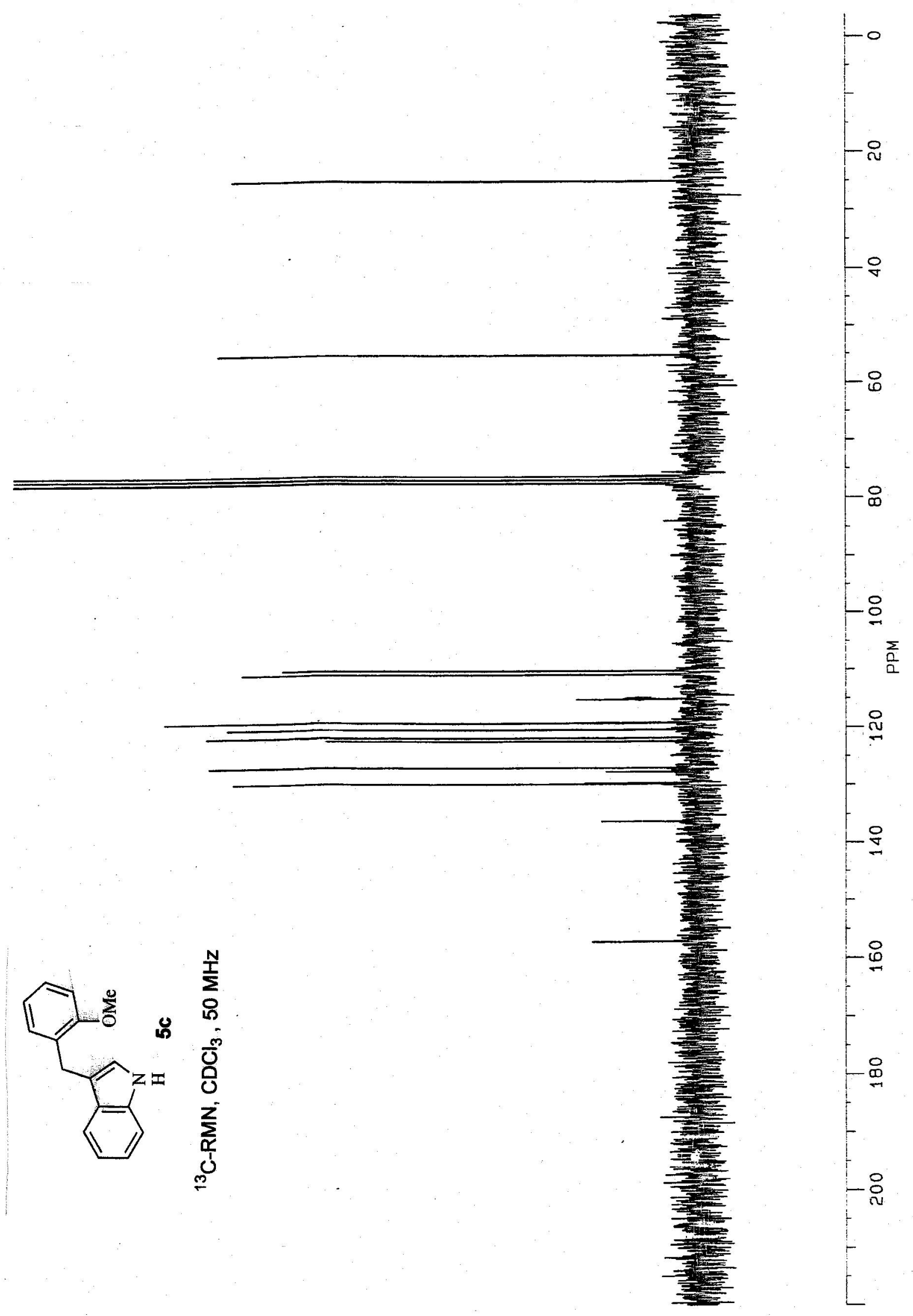




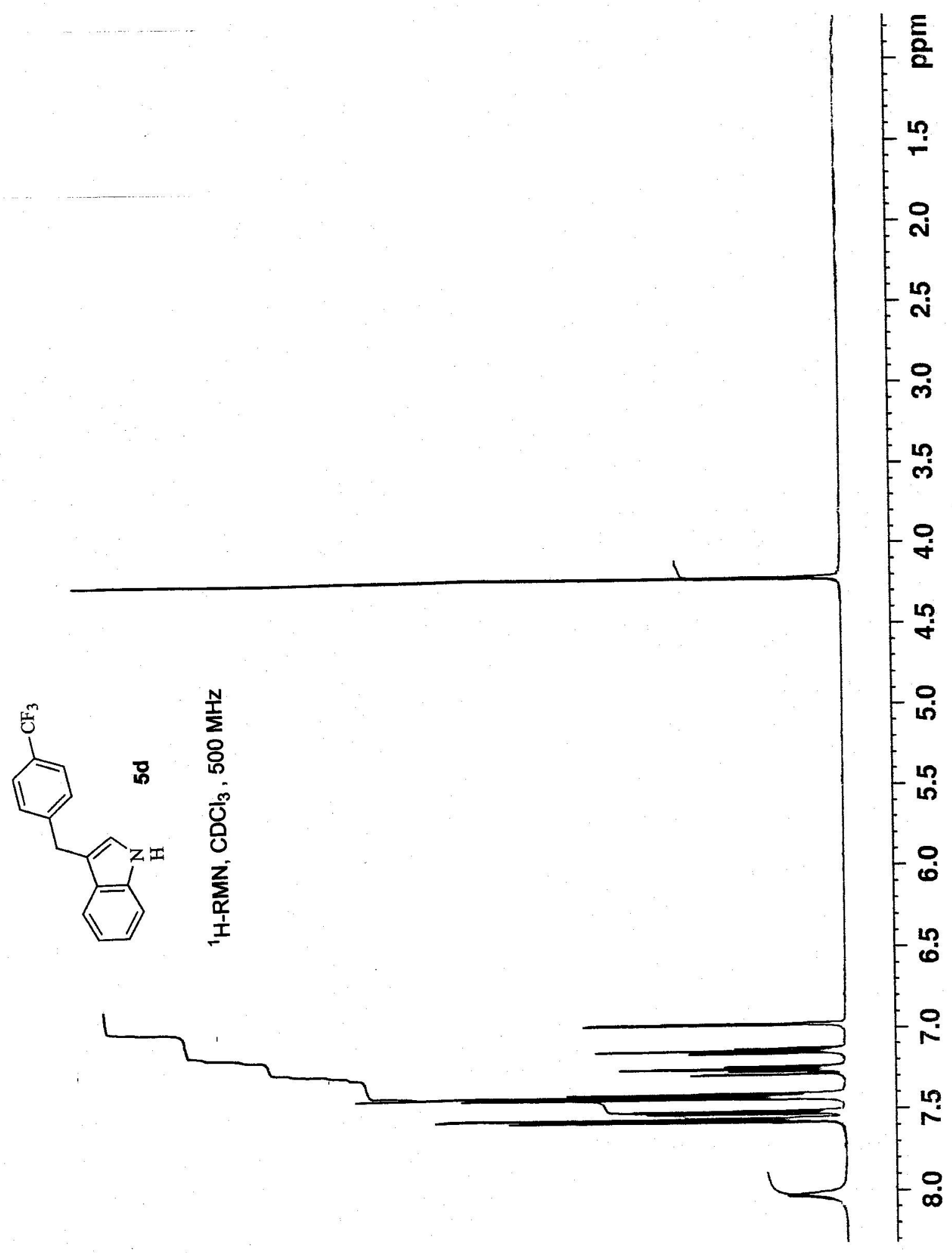




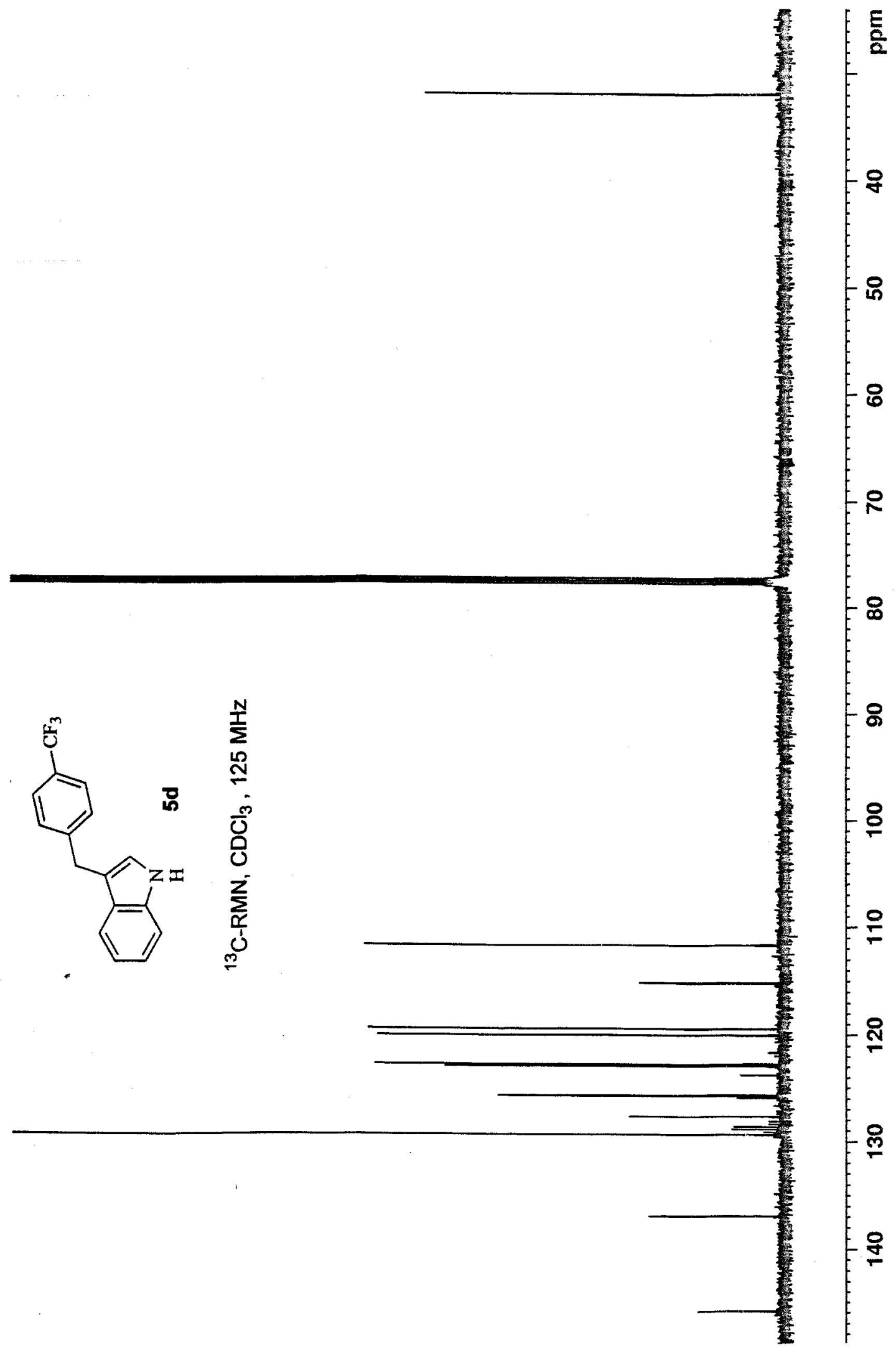




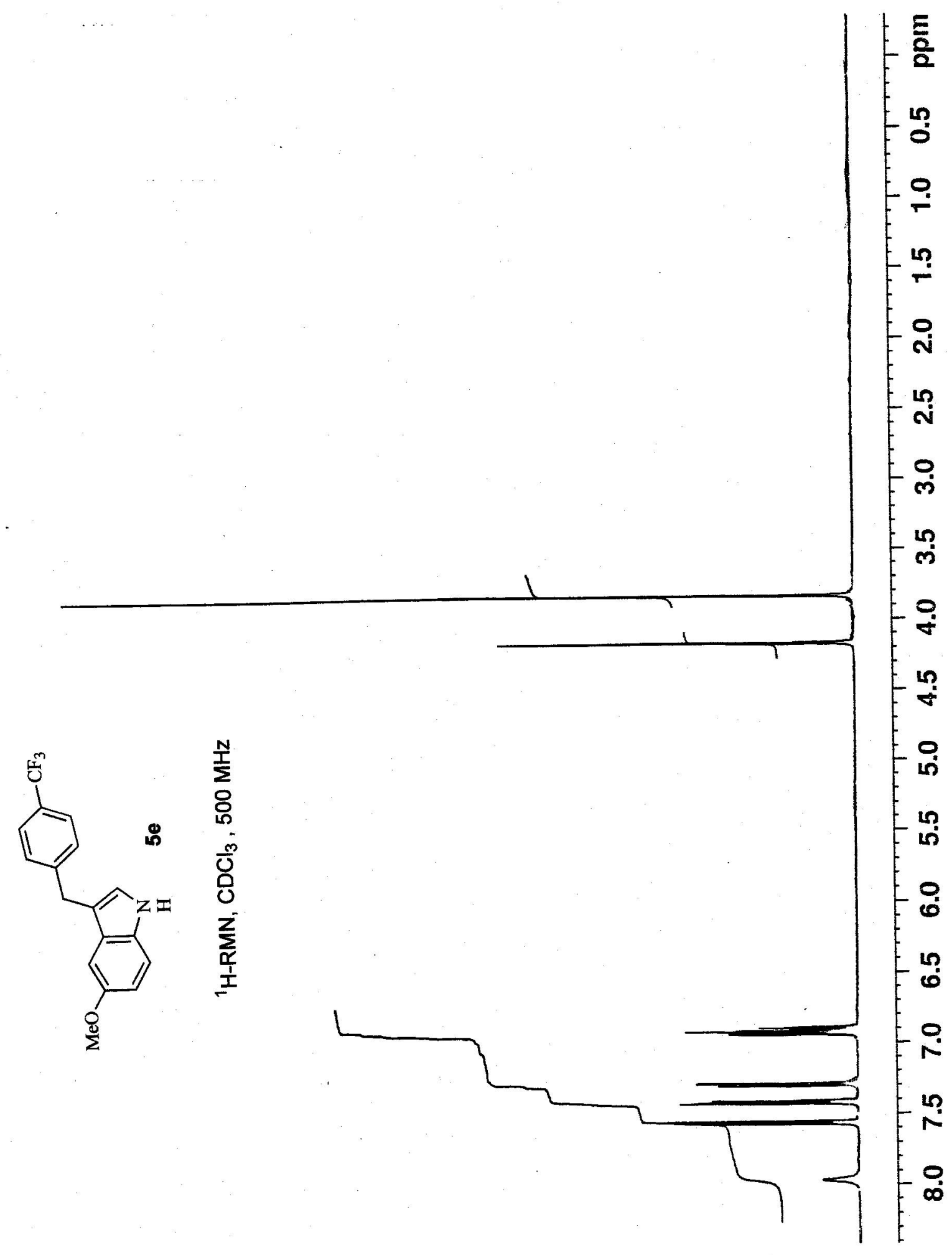




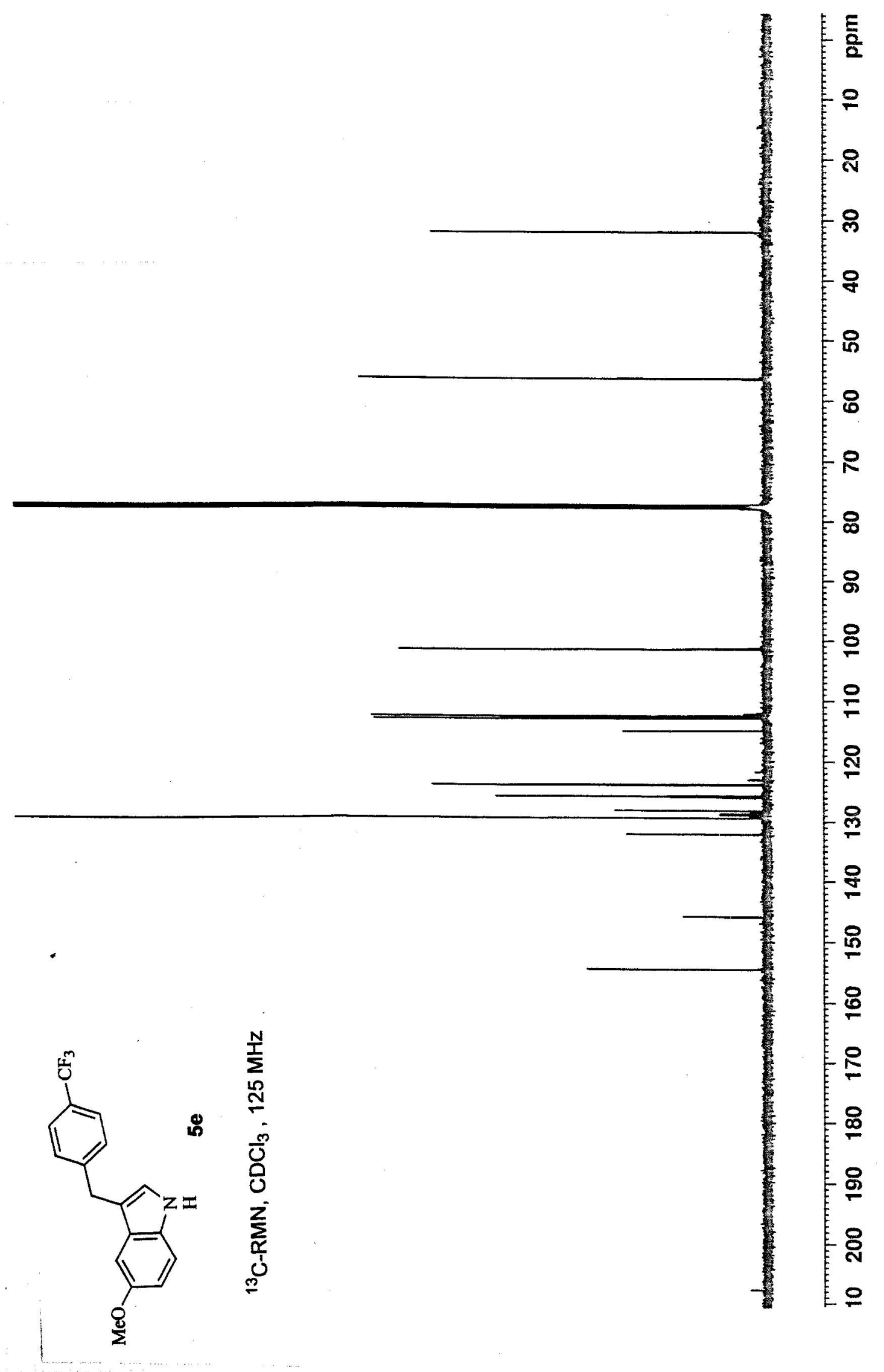




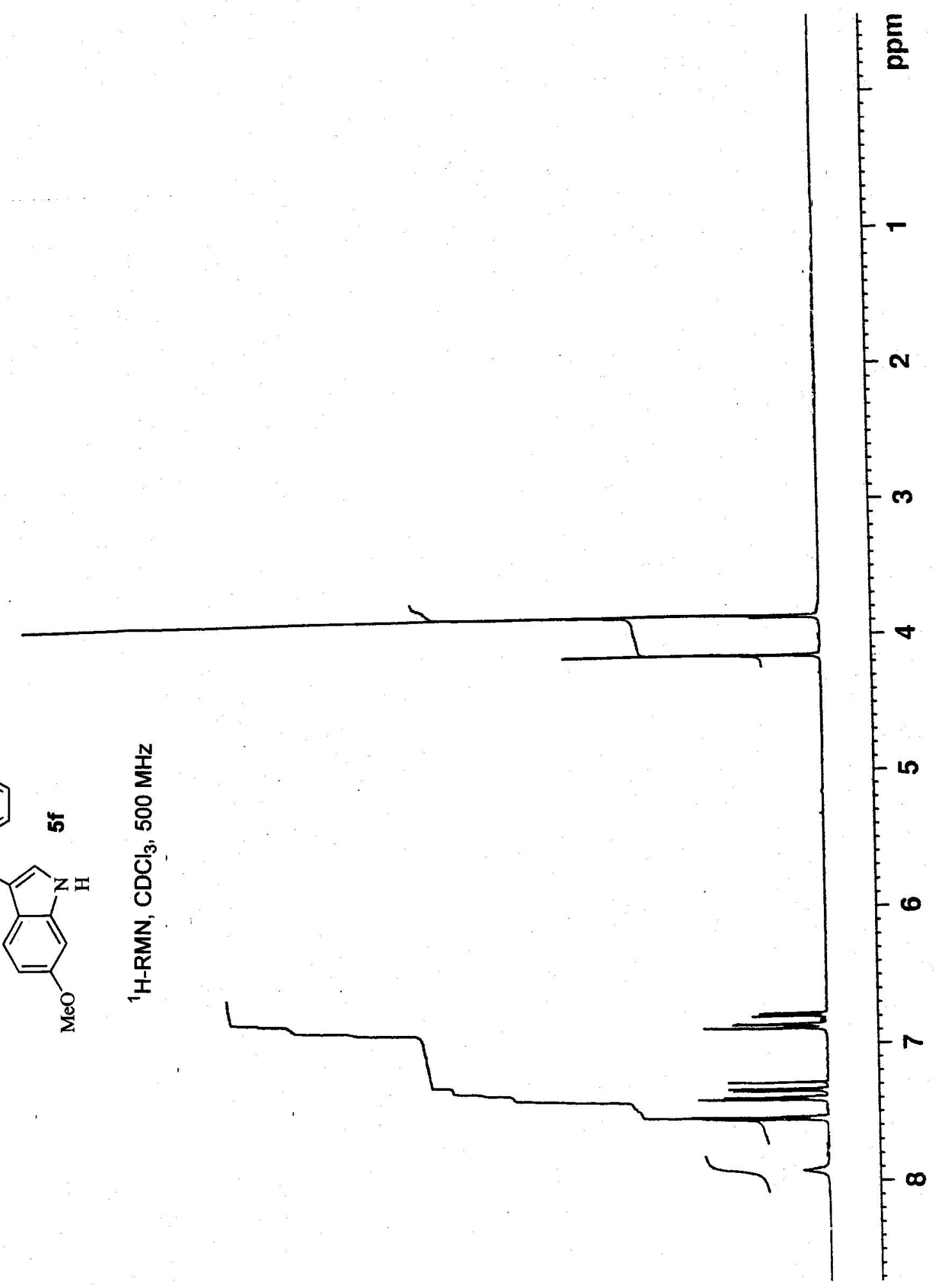




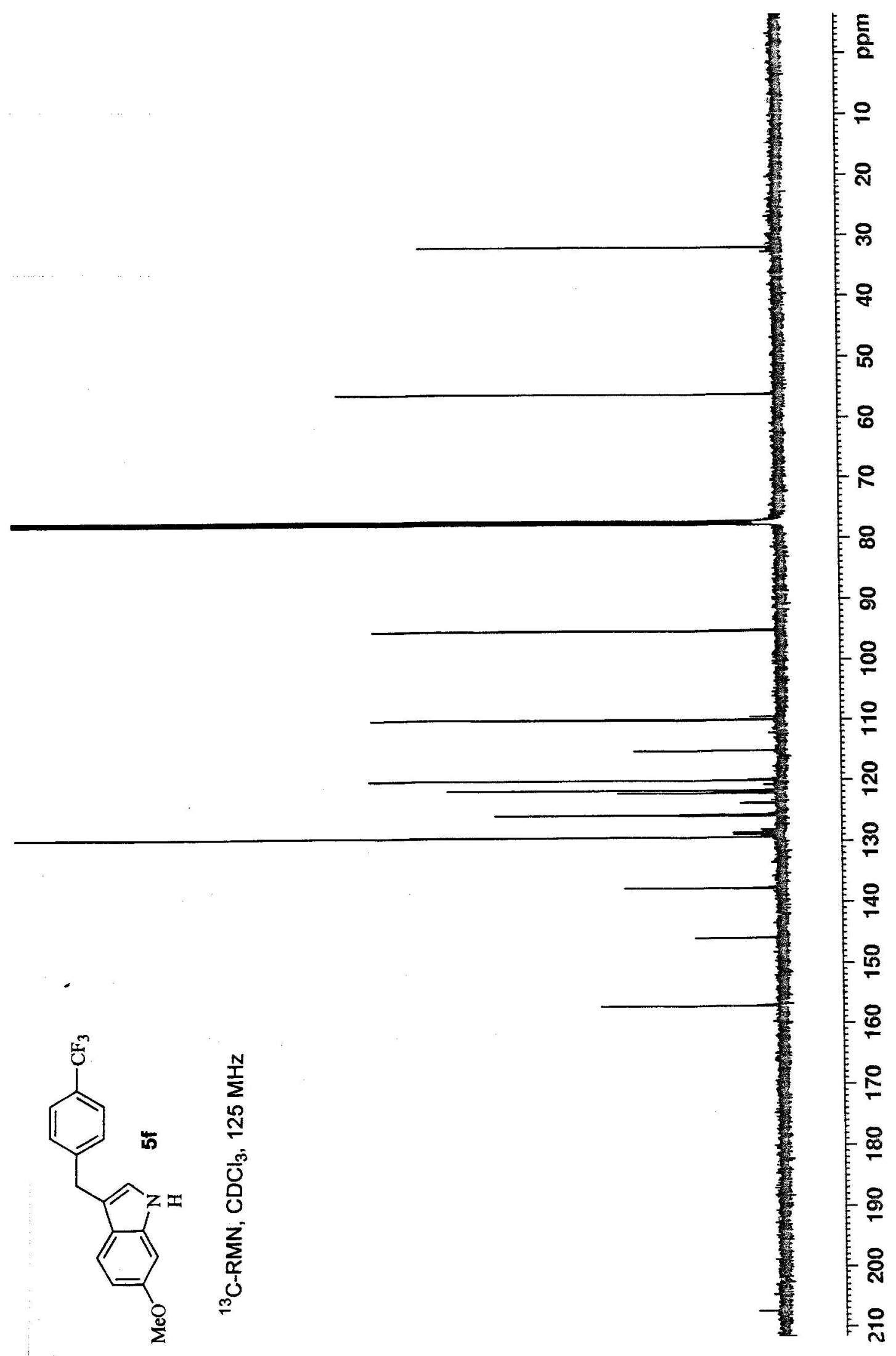




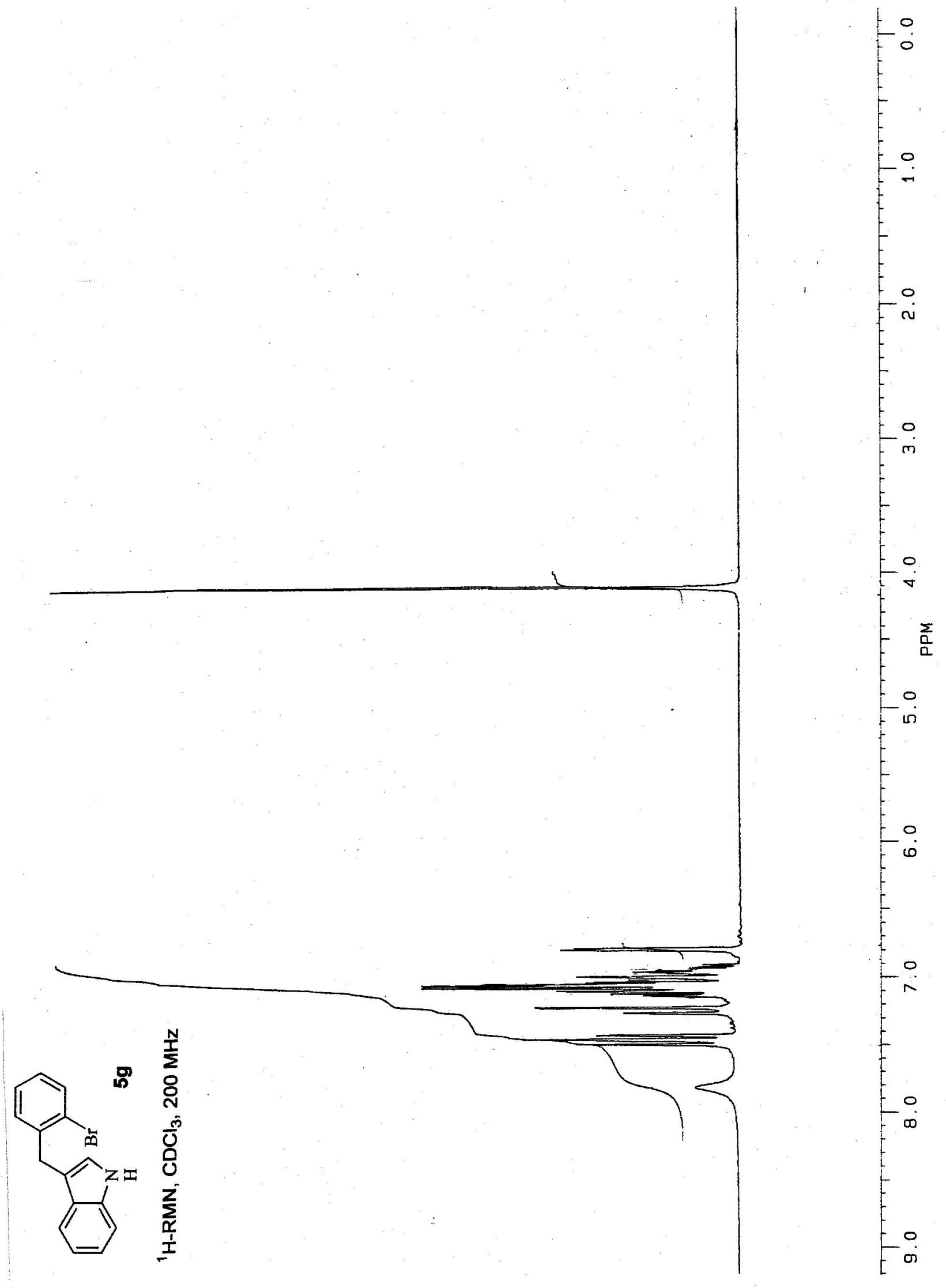




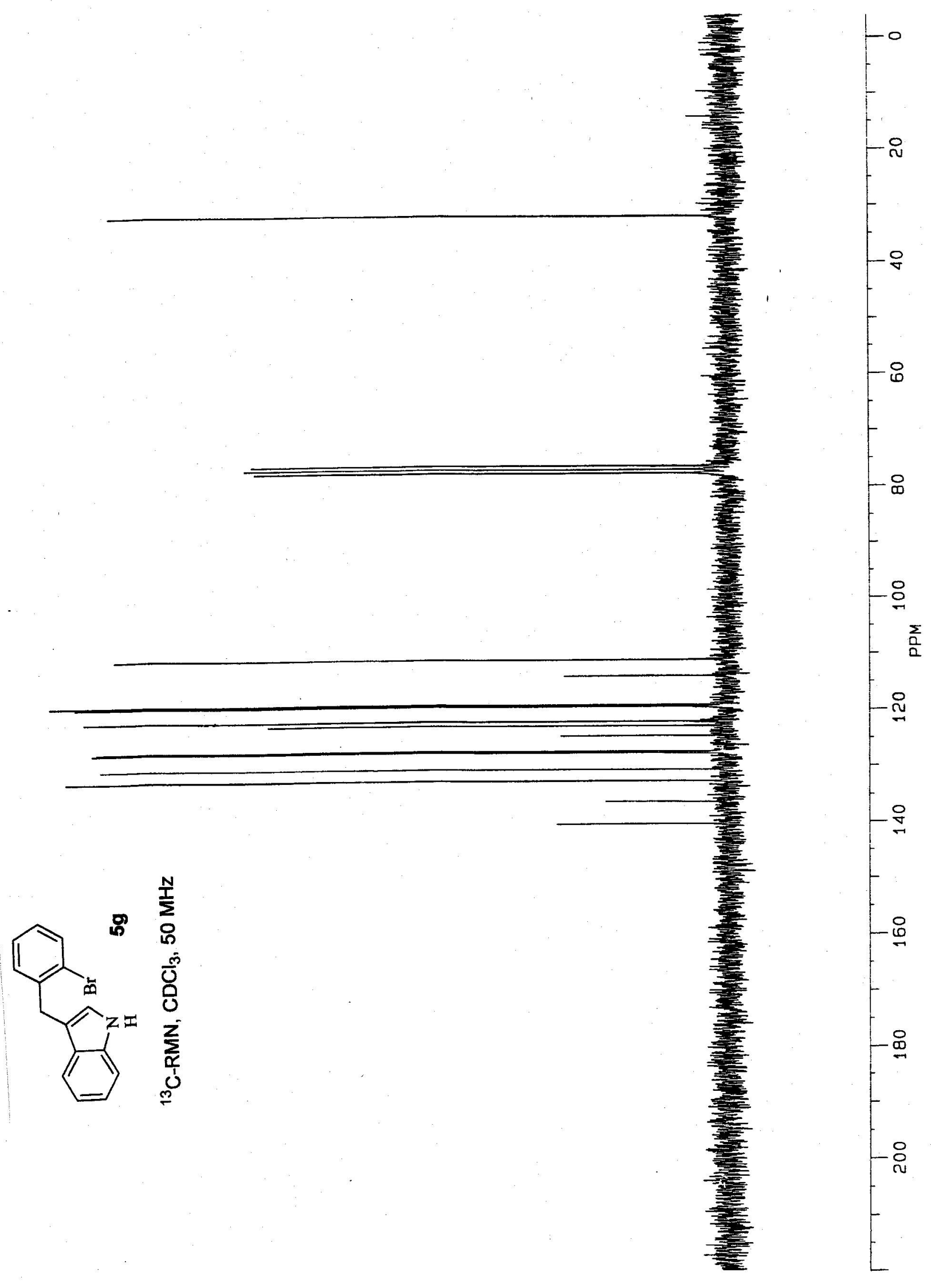




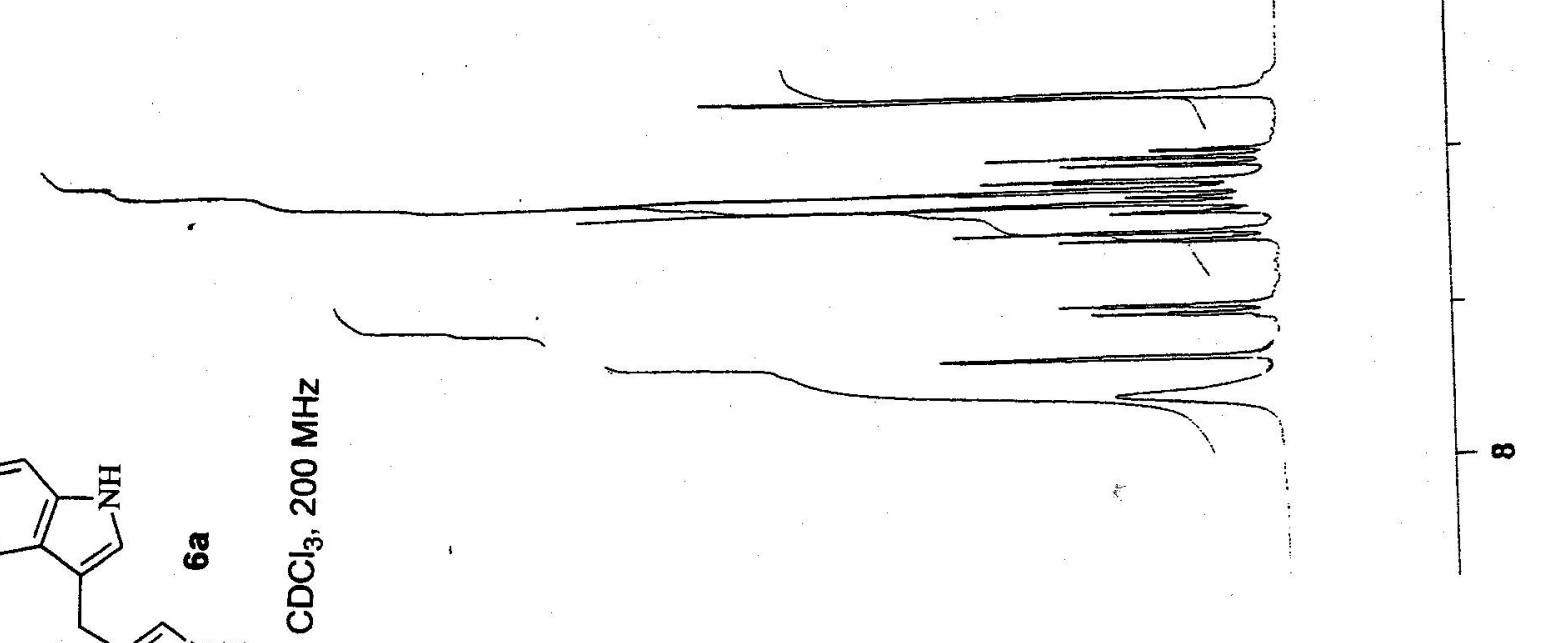

$$
\text { 占 }
$$

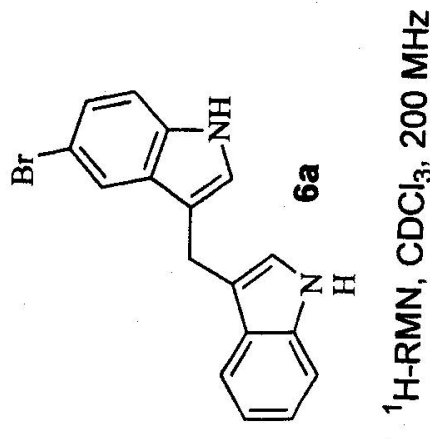




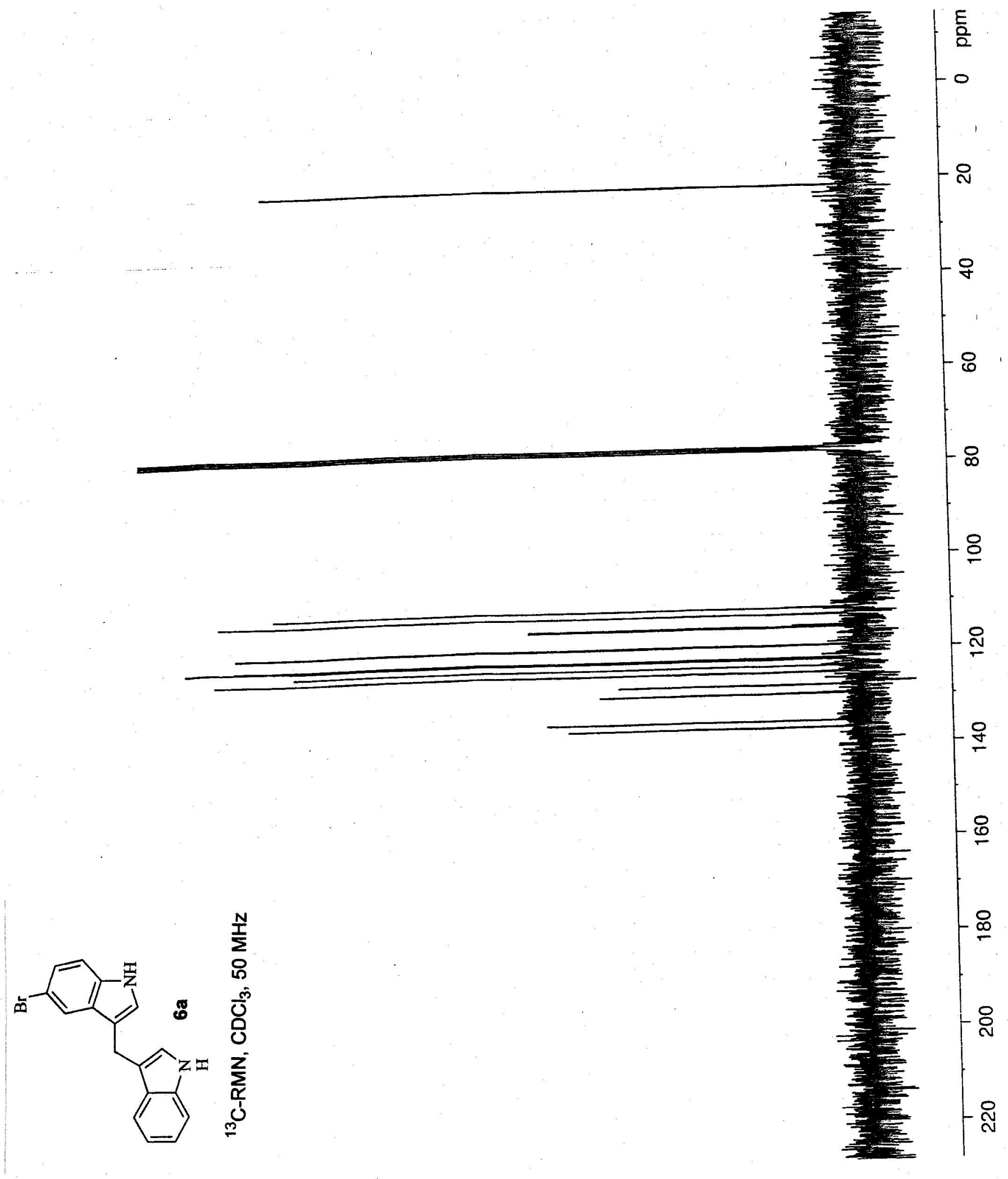




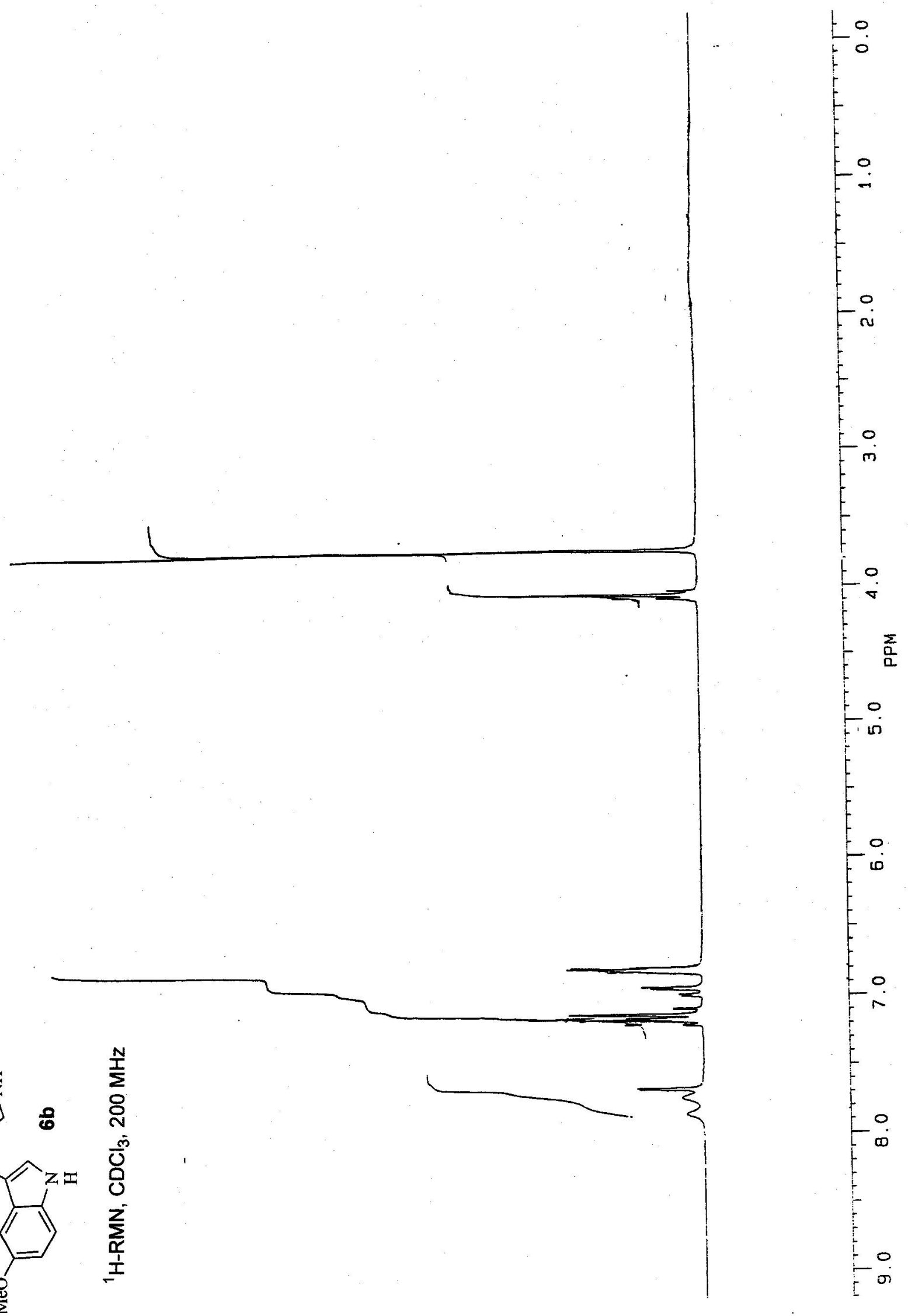




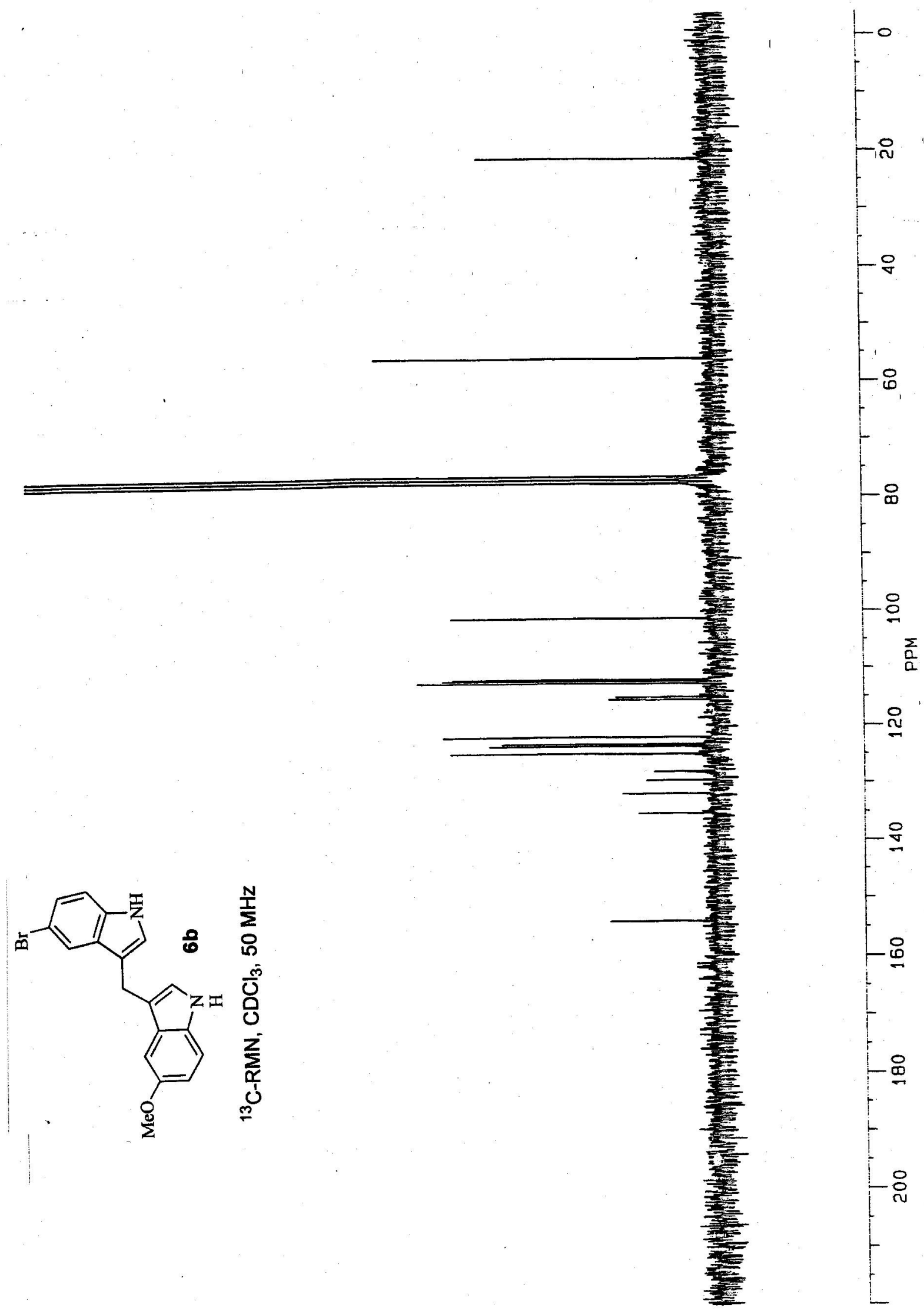



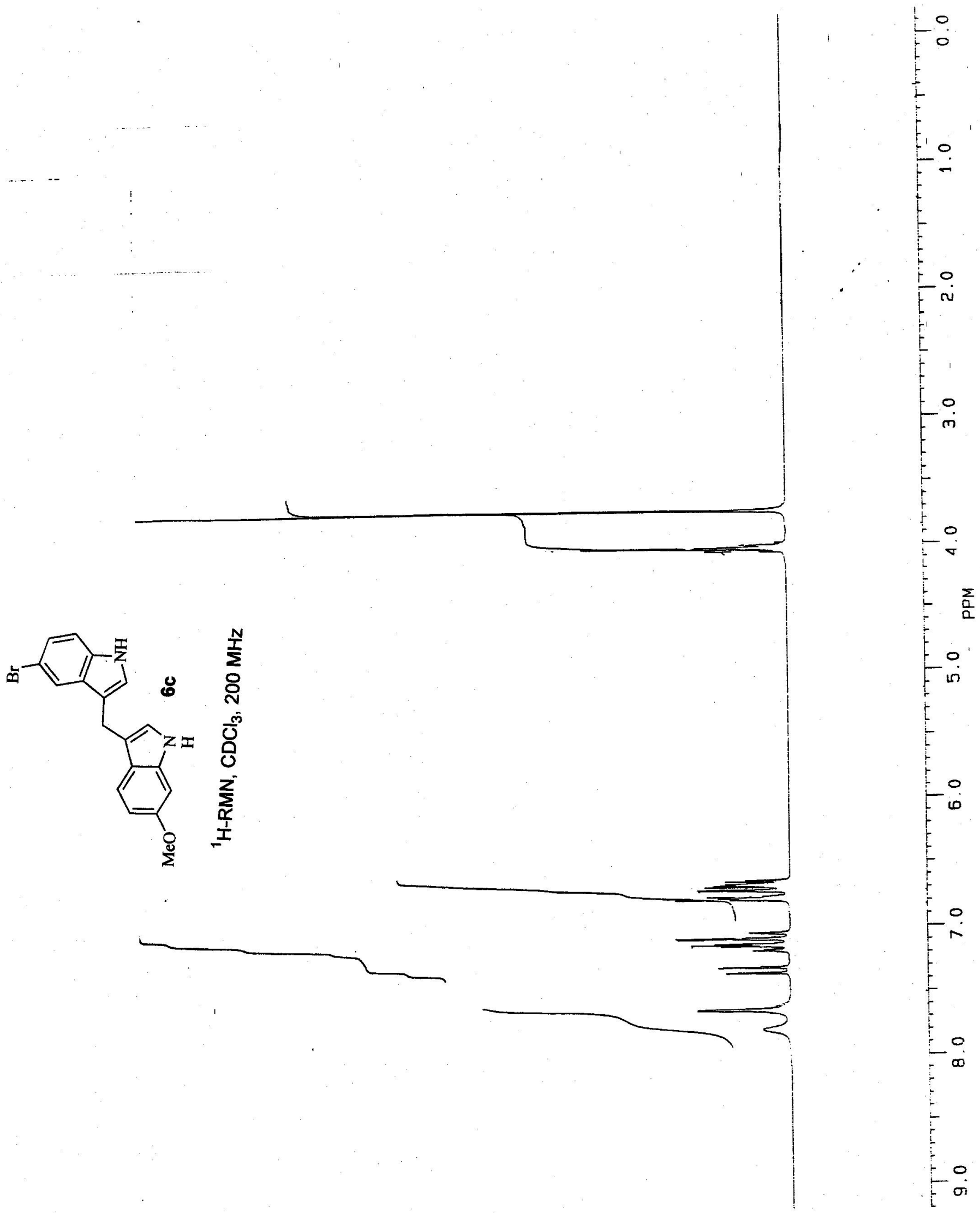


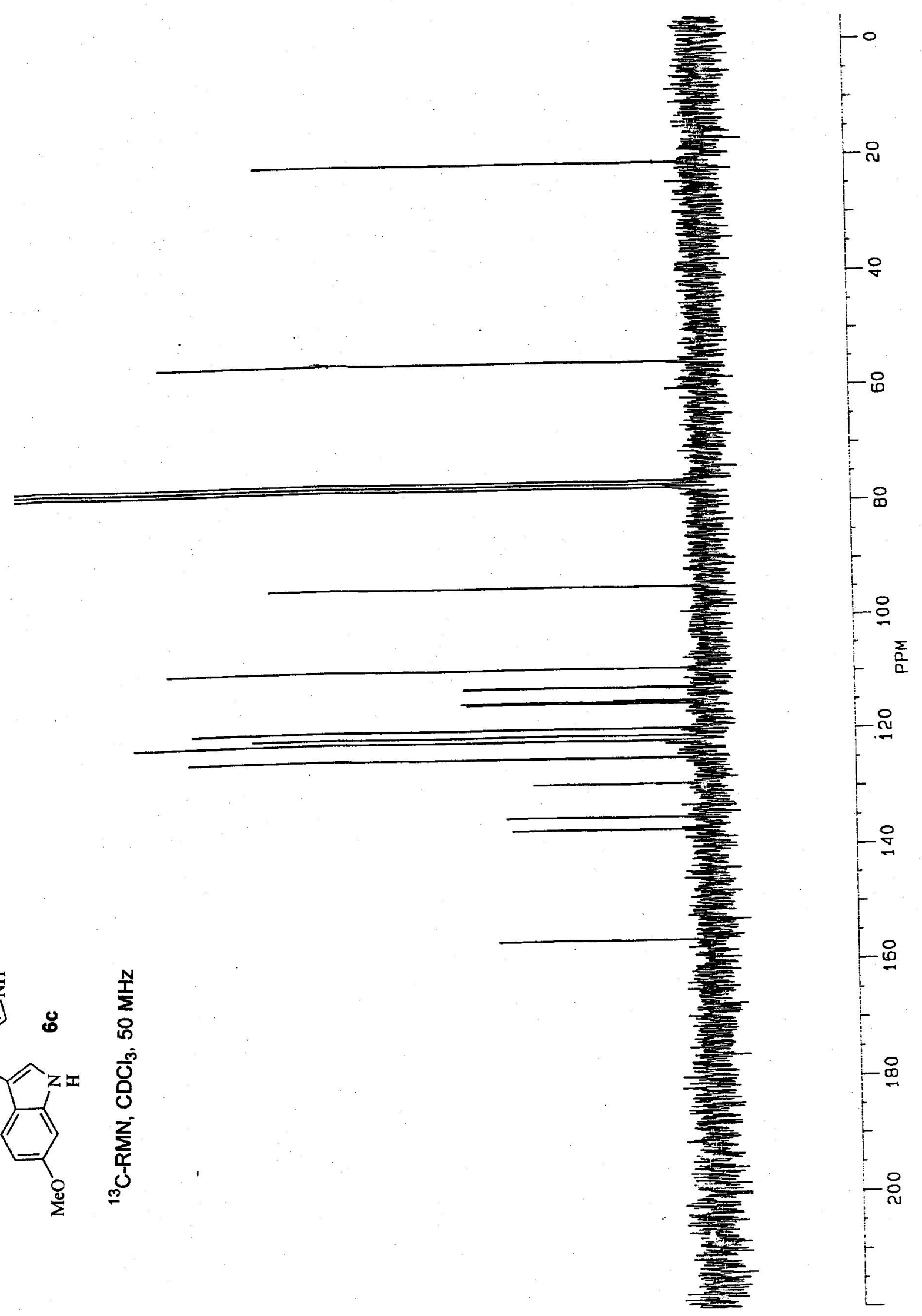




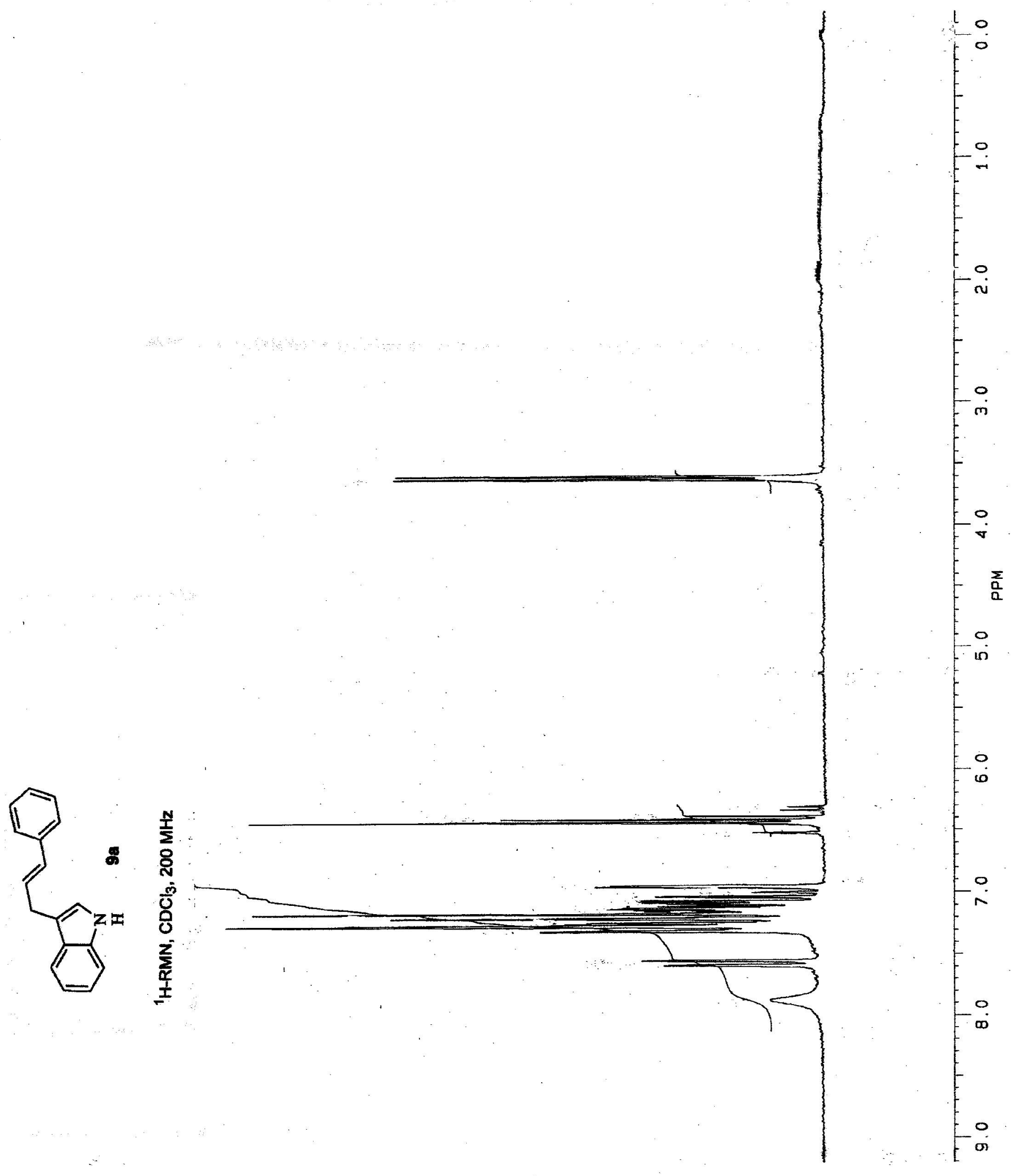




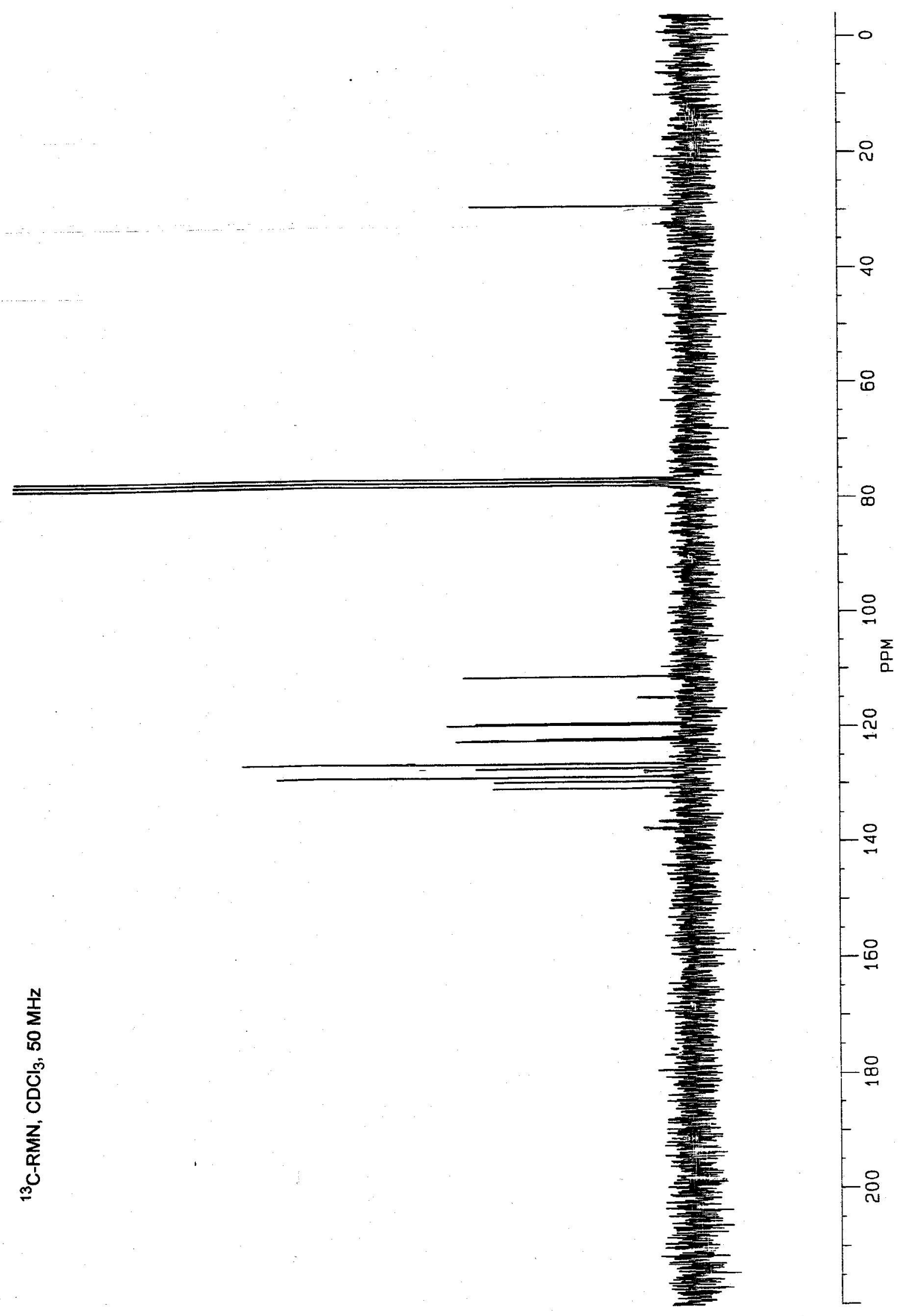




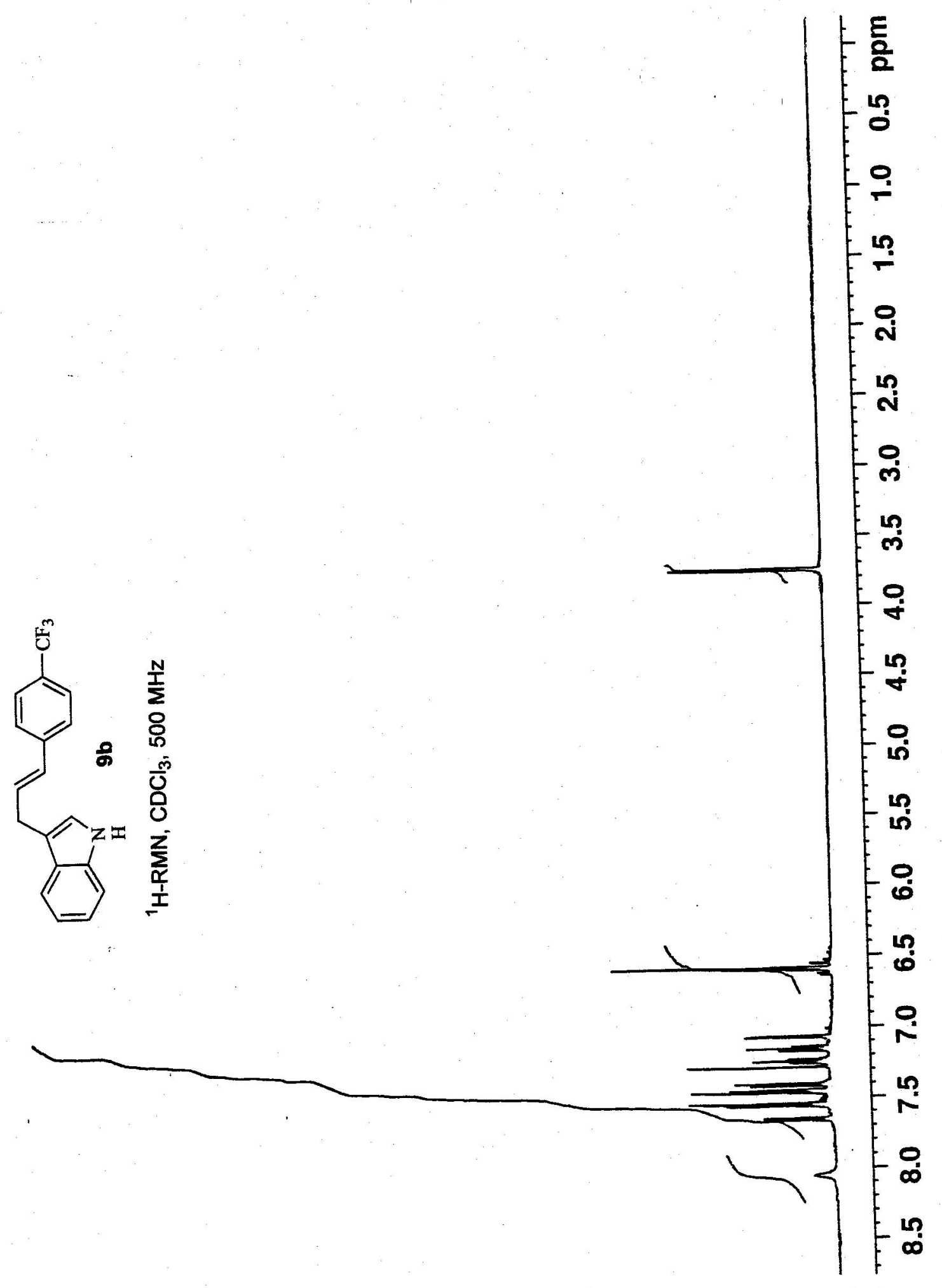




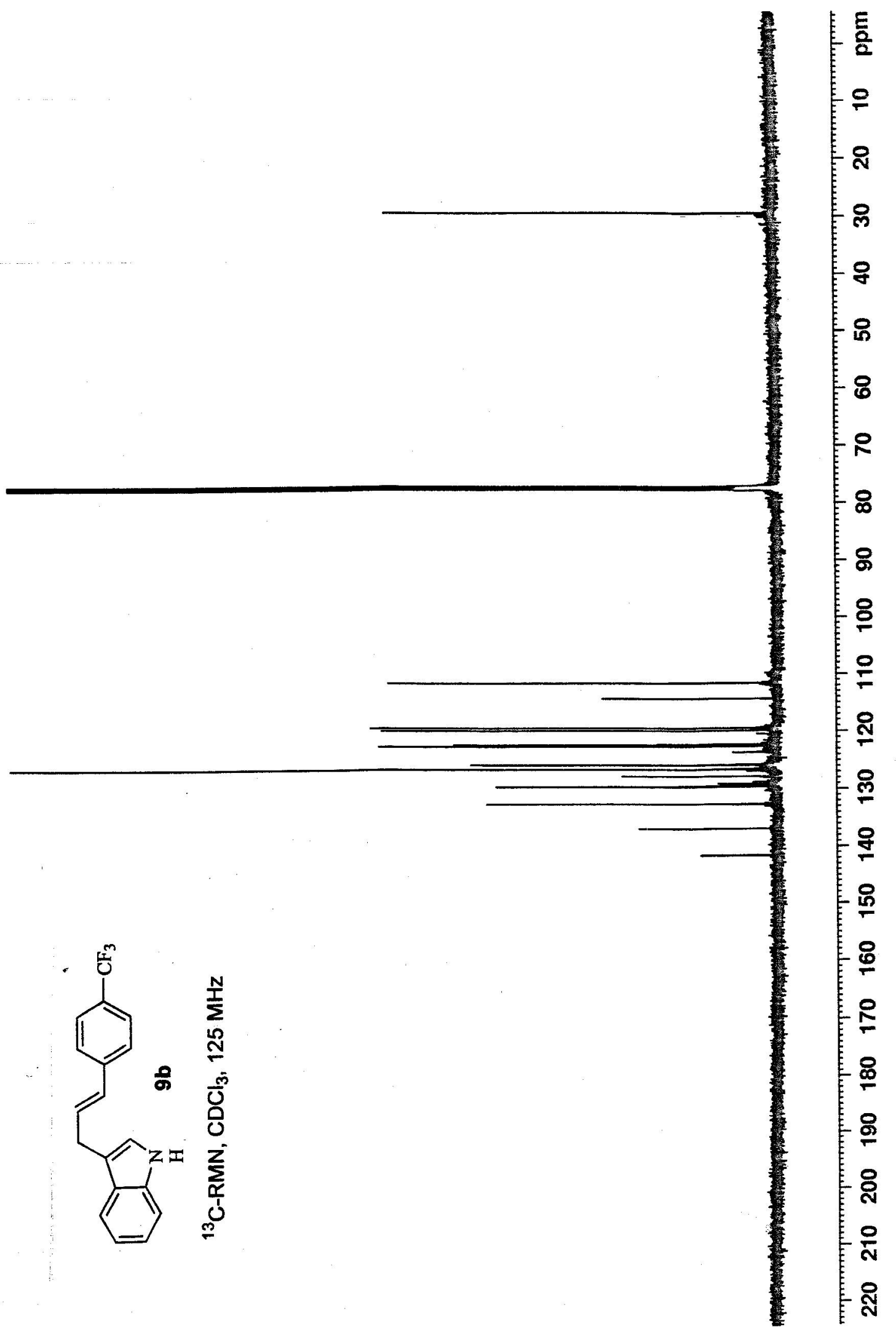




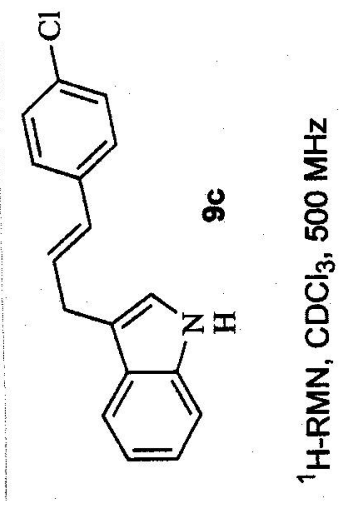




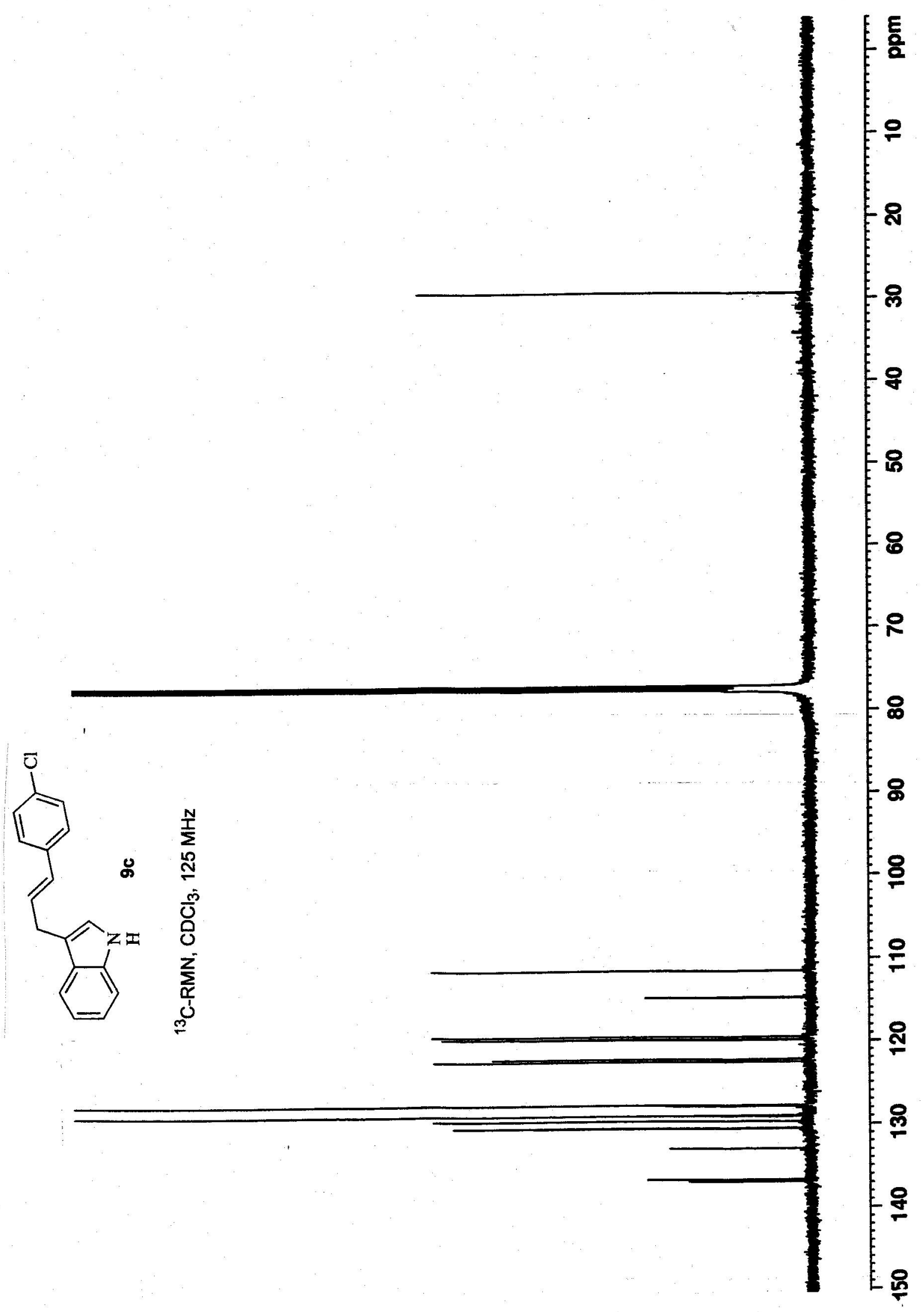



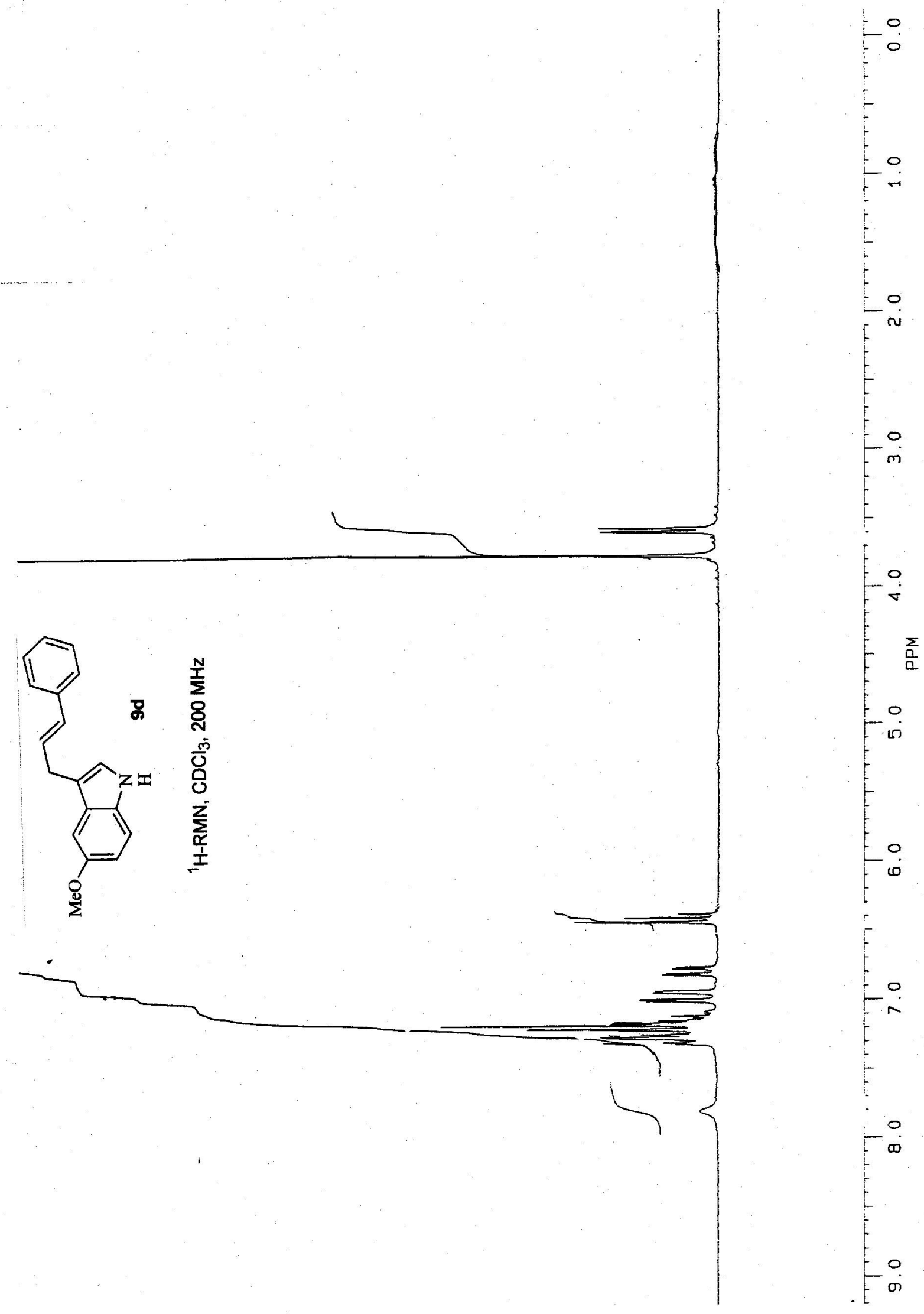


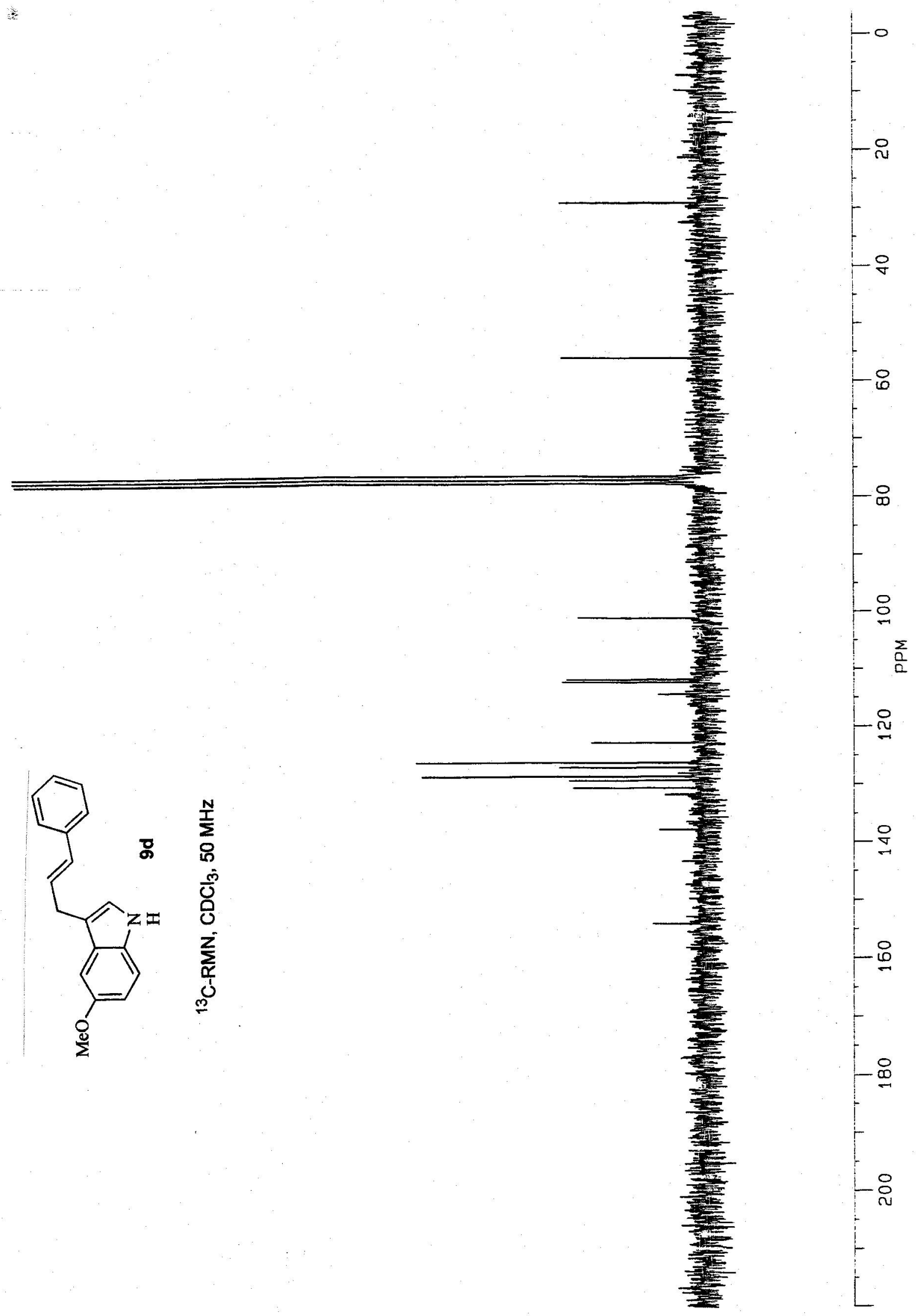




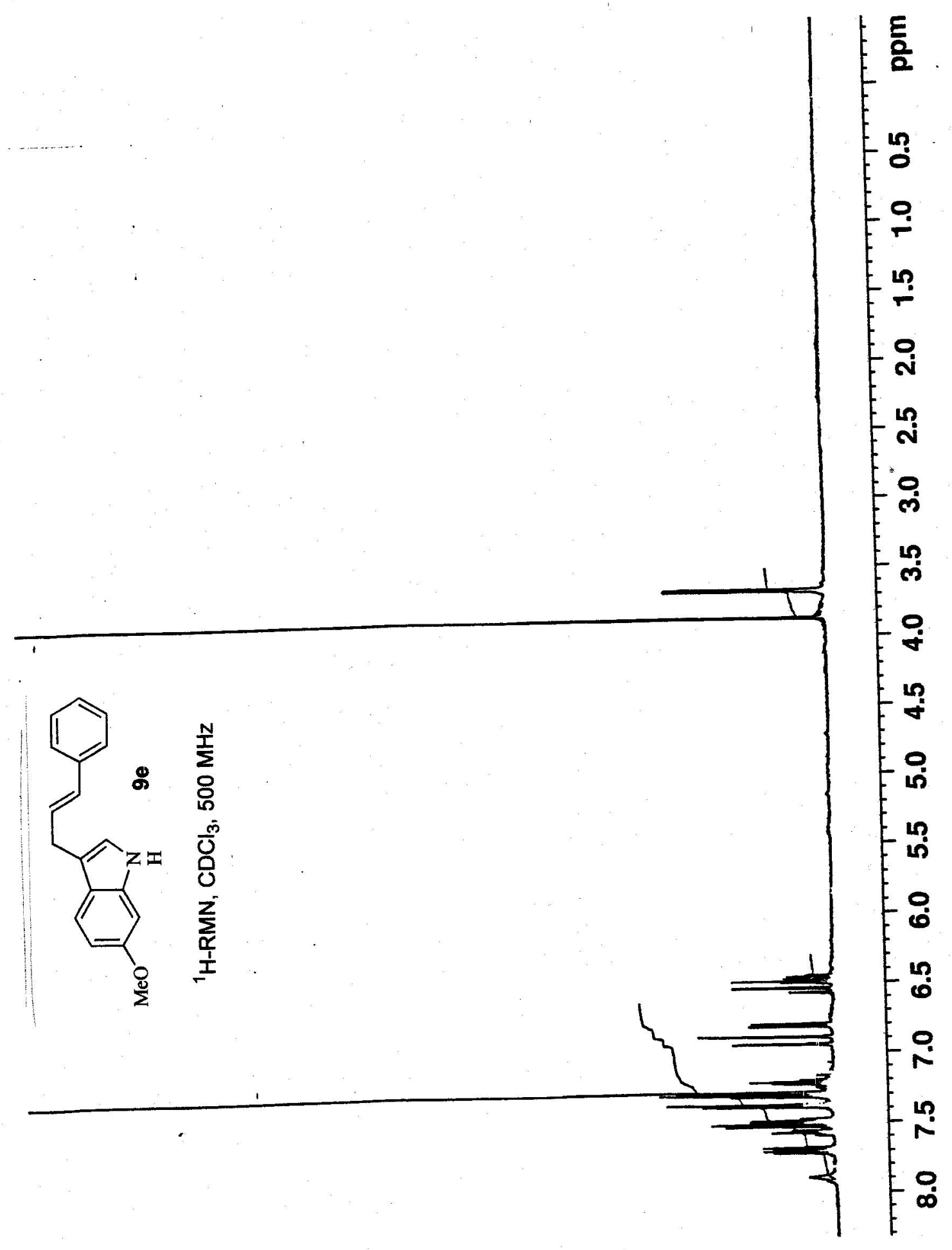




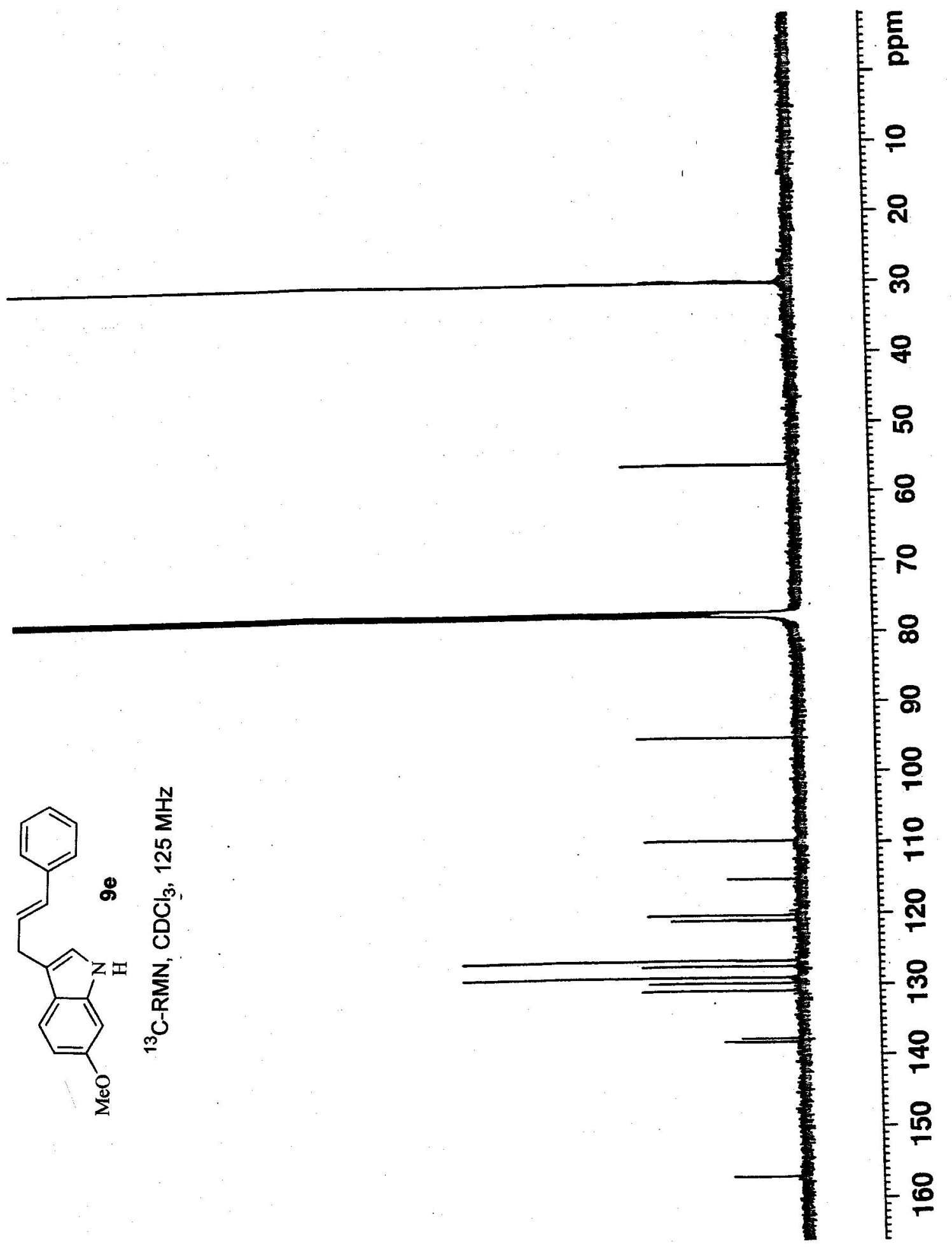

